\title{
ON THE SCATTERING OF TORSIONAL ELASTIC WAVES FROM AXISYMMETRIC DEFECTS IN COATED PIPES
}

\author{
Ray Kirby* \\ School of Engineering and Design, \\ Mechanical Engineering, \\ Brunel University, \\ Uxbridge, Middlesex, UB8 3PH, UK. \\ ray.kirby@brunel.ac.uk
}

Zahari Zlatev

School of Engineering and Design,

Mechanical Engineering,

Brunel University, Uxbridge, Middlesex, UB8 3PH, UK.

\author{
Peter Mudge \\ NDT Technology Group \\ TWI Ltd \\ Cambridge CB21 6AL, UK
}

* Corresponding author 


\begin{abstract}
Long range ultrasonic testing is now a well established method for examining in-service degradation in pipelines. In order to protect pipelines from the surrounding environment it is common for viscoelastic coatings to be applied to the outer surface. These coatings are, however, known to impact on the ability of long range ultrasonic techniques to locate degradation, or defects, within a coated pipe. The coating dissipates sound energy travelling along the pipe, attenuating both the incident and reflected signals making responses from defects difficult to detect. This article aims to investigate the influence of a viscoelastic coating on the ability of long range ultrasonic testing to detect a defect in an axisymmetric pipe. The article focuses on understanding the behaviour of the fundamental torsional mode and quantifying the effect of bitumen coatings on reflection coefficients generated by axisymmetric defects. Reflection coefficients are measured experimentally for coated and uncoated pipes and compared to theoretical predictions generated using numerical mode matching and a hybrid finite element technique. Good agreement between prediction and measurement is observed for uncoated pipes, and it is shown that the theoretical methods presented here are fast and efficient making them suitable for studying long pipe runs. However, when studying coated pipes agreement between theory and prediction is observed to be poor for predictions based on those bulk acoustic properties currently reported in the literature for bitumen. Good agreement is observed only after conducting a parametric study to identify more appropriate values for the bulk acoustic properties. Furthermore, the reflection coefficients obtained for the fundamental torsional mode in a coated pipe show that significant sound attenuation is present over relatively short lengths of coating, thus quantifying those problems commonly encountered with the use of long range ultrasonic testing on coated pipes in the field.
\end{abstract}




\section{INTRODUCTION}

The use of sound waves in the non-destructive testing of oil and gas pipelines is relatively commonplace. The method relies on successfully capturing the sound energy reflected by a remote defect after first launching an incident sound wave along the pipe wall. It is, however, common for pipelines to be coated with a protective material such as bitumen and this is known to significantly affect the propagation of the sound wave. The protective coating is normally viscoelastic and so as the sound wave travels along the pipe the coating acts to dissipate energy, with the effect of progressively lowering the amplitude of both the incident and reflected sound waves as they travel along the pipe. This represents a significant problem in the non-destructive testing of pipelines and serves to restrict the length of pipelines that can be successfully tested using traditional techniques. Here, the influence of a coating on the eigenmodes in a pipe has previously been studied both theoretically and experimentally; however, the experimental data is rather limited and it is generally restricted to modal attenuation over a frequency range much higher than that normally used in the non-destructive testing of pipelines; moreover, there is very little data in the literature that quantifies the influence of the coating on the scattering of sound by a defect. Accordingly, this paper aims to quantify the influence of viscoelastic coatings on sound propagation in a finite section of pipe; both measurement and prediction are used to analyse the scattering of sound waves from defects in a coated pipe over a frequency range normally found in the long range ultrasonic testing of pipelines, which is approximately $20-140 \mathrm{kHz}$.

It is well known that a viscoelastic medium dissipates energy within a travelling elastic wave. For example, Chan and Cawley [1] studied the effect of viscoelasticity on the dispersion curves for a high density polyethylene pipe. Energy dissipation was included by adding an imaginary component to the Lamé constants, although modal attenuation was studied only for Lamb modes 
in a plate. Barshinger and Rose [2] extended the work to the analysis of a number of viscoelastic layers and published dispersion curves for a steel pipe coated with epoxy. Both Chan and Cawley [1] and Barshinger and Rose [2] use analytic techniques to derive the governing dispersion relation, and Barshinger and Rose later discuss the problems this presents when it comes to finding the roots of this equation. Here, the addition of a viscoelastic layer means that the roots of the dispersion relation are complex and it is much harder to locate these roots in the complex plane (see also Simonetti and Cawley [3] for a discussion on coated plates). In view of the problems with root finding, recent work has focussed on using numerical techniques such as the finite element method to locate the roots of the governing dispersion relation. For example, Bartoli et al. [4] use the finite element method to solve the dispersion relation for a waveguide of arbitrary cross-section, as well as for a viscoelastic plate. Bartoli et al. also provide a detailed discussion on the background to this finite element based method, which is referred to in the elastic waveguide literature as the Semi Analytic Finite Element (SAFE) method. Of course, this method is not limited to elastic waveguides and it has been present in the acoustic waveguide literature for many years, although here the SAFE nomenclature has not been adopted. Relevant examples of the "SAFE" method in the acoustic waveguide literature include Astley and Cummings [5] and Kirby [6], since both articles show how energy dissipation is added to an acoustic waveguide through the use of complex material properties. The SAFE method is popular because it solves the dispersion relation without recourse to root finding and it works equally well with complex roots, it is therefore ideally suited to the study of coated pipelines.

The SAFE method has seen extensive use in the elastic waveguide literature in recent years (see for example [7-10]). The use of this method to study energy dissipation is, however, less well reported and it is only recently that sound attenuation has been included in the SAFE method for elastic waveguides. For example, Castaings and Lowe [11] calculate the eigenmodes for an elastic waveguide of arbitrary cross section surrounded by an absorbing region. Energy 
dissipation in coated pipes was studied by both $\mathrm{Mu}$ and Rose [12] and Marzani et al. [13], who both used the SAFE method. Here, the SAFE method is applied in its most general form, that is, the problem is solved for all available symmetrical and non-symmetrical eigenmodes (the number of independent eigenmodes found being equal to the number of nodes in the finite element mesh). This general methodology is readily applied using generic finite element based software in which one enters the general mass and stiffness matrices for the problem. However, a disadvantage with this approach is that the solution of the governing dispersion relation delivers an unordered list of modes and one cannot readily distinguish between each type of mode. Mu and Rose [12] note this problem and derive an orthogonality relation in order to sort each mode type. Their method relies on solving the dispersion relation at two frequencies that are sufficiently close to one another so that one can make use of the orthogonality relation, and $\mathrm{Mu}$ and Rose [12] show that this can be used successfully to sort eigenmodes for a coated pipe. However, in general it is possible that very small frequency increments may be required in order to successfully sort modes, especially for a coated pipe, and so this method is potentially computationally expensive.

The SAFE method has been shown to be a reliable method for finding the eigenmodes of a coated pipe, but the method does not provide the amplitudes of each mode. The computation of modal amplitudes implies the study of a finite length of pipe with an applied forcing. One can, of course, use numerical methods to discretise the entire (finite) pipe length and this approach was used by Hua and Rose [14] in the study of coated pipes. Hua and Rose used the commercial software package ABAQUS in order to compare modal attenuation using a full finite element discretisation of the pipe with predictions obtained using the SAFE method. Hua and Rose demonstrate reasonably good agreement between the two predictions, although it is inevitable that for long pipes the ABAQUS model will be computationally expensive. Moreover, in order to represent the pipe coating, Hua and Rose found it necessary to infer values for the "damping 
coefficients" required by the commercial software and so it is possible that this is the reason for the discrepancy between the two models (which in principle should deliver identical attenuation predictions). In order to try and reduce computational expenditure, Predoi et al. [15] used the SAFE method to find the modal characteristics for a viscoelastic plate and then coupled this to a modal projection method, based on modal orthogonality, in order to recover the amplitudes of the modes found using the SAFE method. Essentially, the method couples a modal expansion to a full finite element discretisation of the problem close to a defect and here Predoi et al. studied a notch (or "trough") in a rectangular viscoelastic plate. This method has the potential to be much more computationally efficient than the method used by Hua and Rose [14], since it reduces the size of the region that needs to be meshed, although it is interesting to note that Predoi et al. still required 147,744 degrees of freedom at $200 \mathrm{kHz}$.

Clearly it is sensible to develop computationally efficient ways of modelling long lengths of pipe, especially as the advantage of non-destructive testing is that one can, in principle, examine long lengths of pipe. Here, the true power of the SAFE method lies not just in calculating the eigenmodes but in using these modes to model long uniform waveguides of arbitrary cross section. Providing one uses an appropriate technique to analyse scattering from an area discontinuity (or defect), the modal amplitudes for long pipe lengths can readily be calculated. There are two ways in which to do this: (i) if the defect has a uniform cross section then one can use a modal expansion for the defect as well; or (ii) if the defect is non uniform then one may use a finite element discretisation for the region surrounding the defect, adopting an approach similar to that described by Predoi et al. [15] when studying plates. Both approaches seek to use only modal expansions for uniform regions, one then applies a finite element discretisation only when absolutely necessary in order to minimise computational expenditure. After application of the appropriate continuity conditions over each area discontinuity (defect) one may then readily recover the modal amplitudes. This approach has been used by the first author in the study of 
sound dissipation in acoustic waveguides; for example, Kirby used point collocation [6] and mode matching [16] to enforce acoustic pressure and velocity matching conditions over two area discontinuities, after first finding the eigenmodes using a SAFE method. For non uniform regions, Kirby $[16,17]$ adopts a hybrid method in which a full finite element discretisation of a non uniform section is matched to the surrounding (uniform) duct using either point collocation or mode matching. It is shown in these papers that these methods are potentially very computationally efficient and can be used to study relatively large systems and/or high frequencies.

Of course, mode matching and hybrid methods are not limited to the acoustic waveguide literature and these have been applied to many problems in elastic waveguides. For example, Engan [18] used mode matching to study elastic wave propagation in a rod, Hayashi [10] and Demma et al. [19] studied steps and notches in a plate, and mode matching was applied to a pipe by Ditri and Rose [20] and Vogt et al. [21]. For the hybrid method, relevant examples include Predoi et al. [15] and Imhof [22], who studied elastic wave propagation in plates; the scattering from non uniform defects in pipes is examined by Hayashi [10], Zhuang et al., [23], Bai et al. [24] and Zhou et al. [25]. However, it is only relatively recently that the SAFE method has been used as the basis for these numerical techniques, see for example Hayashi [10] and Zhou et al. [25], and there are no studies which use the SAFE method to analyse scattering from defects in coated pipes. Accordingly, this paper uses the SAFE method to find the eigenmodes for the uniform regions of a pipe, and then applies either a mode matching or hybrid method to analyse scattering from a defect in a coated pipe. Since this method has not been applied before to coated pipes, the method is first validated for the relatively straightforward problem of axisymmetric sound propagation in a coated pipe with an axisymmetric defect. Moreover, the analysis is limited here to the propagation of the fundamental torsional mode - the so called $\mathrm{T}(0,1)$ mode. The advantage of limiting this study to the scattering of the $\mathrm{T}(0,1)$ mode from an axisymmetric 
defect is that one may then readily compare predictions against theoretical and experimental data reported in the literature for an uncoated pipe (see for example [26-29]). Furthermore, this also facilitates comparison with experimental measurements since it is relatively straightforward to generate and measure the $\mathrm{T}(0,1)$ mode and at the same time to minimise coherent noise.

A further reason for restricting experimental measurements to the $T(0,1)$ mode is that there is a lack of experimental data in the literature for the bulk acoustic properties of typical coating materials. Those papers published on the SAFE method for bitumen coated pipes draw on the data measured by Barshinger and Rose [2]; however, there is a question mark over the accuracy of this data since these properties are inherently difficult to measure over the entire frequency range of interest. This has necessitated the extrapolation of data measured at frequencies above 1 $\mathrm{MHz}$ to those lower frequencies normally of interest in long range ultrasonic testing. Barshinger and Rose did investigate this by measuring modal attenuation in a coated pipe and comparing this to predictions of attenuation using their measured bulk acoustic properties; however, agreement between prediction and measurement is limited and there is very little data reported below 1 MHz. Moreover, the bulk shear properties are known to be especially difficult to measure accurately and so it appears sensible first to isolate this parameter and to investigate the comparison between measurement and prediction for the fundamental torsional mode. Accordingly, this article will focus on measuring and predicting elastic wave propagation at lower ultrasonic frequencies, in the range $20-120 \mathrm{kHz}$. The study of axisymmetric defects will also enable the use of well established experimental methods previously reported for measuring the reflection coefficients in uncoated pipes [29].

In Section 2, this article begins by using the SAFE method to obtain only the torsional modes for a coated pipe. This will deliver a reduced system of equations for the torsional modes and avoids the need to sort torsional modes from other modes such as longitudinal and flexural. The SAFE 
method is then utilised in a mode matching algorithm, which is reported in Section 2.1, and a hybrid formulation reported in Section 2.2. In Section 4 predictions are compared against experimental data obtained using the methodology described in Section 3. Here, the theoretical predictions are validated first by comparison against a simple "plane wave" analytic formulation for an uncoated pipe, as well as against data published in the literature. After validation of the experimental and theoretical method for an uncoated pipe, the same techniques are then used to analyse a coated pipe.

\section{THEORY}

The propagation of elastic waves in a pipe is governed by Navier's equation [30],

$$
\mu_{\mathrm{s}, \mathrm{c}} \nabla^{2} \mathbf{u}^{\prime}+\left(\lambda_{\mathrm{s}, \mathrm{c}}+\mu_{\mathrm{s}, \mathrm{c}}\right) \nabla\left(\nabla \cdot \mathbf{u}^{\prime}\right)=\rho_{\mathrm{s}, \mathrm{c}} \frac{\sim \cdots}{\partial t^{2}}
$$

where ${ }^{\mu}$ and $\lambda$ are the Lamé constants, $\mathbf{u}^{\prime}$ is the displacement vector, $\rho$ is the density of the pipe wall and $t$ is time. It is assumed here that the pipe consists of a steel substrate in which sound attenuation may be neglected, coated externally by a material in which sound attenuation is assumed to take place. Here, the steel substrate is denoted by subscript s, and the external coating

by subscript $\mathrm{c}$. It is convenient here to use using a cylindrical co-ordinate system (say, $r, \theta$ and z), and for an axisymmetric problem this delivers three equations, only two of which are coupled. Thus, for an axisymmetric problem only, the two coupled equations (displacements in the $r$ and $z$ direction) govern the propagation of the so-called longitudinal pipe modes, whereas the third equation (displacement in the $\theta$ direction) governs the propagation of the so-called torsional modes. This article will focus only on the torsional modes, and so Navier's equation simply reduces to a scalar equation of the form: 


$$
\overline{c_{\mathrm{s}, \mathrm{c}}^{2}} \frac{\overline{\partial t^{2}}}{\mathrm{v}} \mathrm{v} v=\mathrm{u}
$$

where $v^{\prime}$ is the wall displacement in the $\theta$ direction, and $C_{\mathrm{s}}$ and $C_{\mathrm{c}}$ are the torsional bulk wave velocities in the pipe wall (substrate) and the pipe coating, respectively.

\subsection{Eigenvalue Analysis}

An eigenvalue problem is obtained by first expanding the displacement $v^{\prime}$ over the pipe eigenmodes to give

$$
v(r, z ; t)=\sum_{n=0} v *(r) e^{-\cdots \cdots \cdots \cdots},
$$

where $v$ is a modal eigenvector, $k_{\mathrm{s}}=\omega / c_{\mathrm{s}}$, so that $\lambda$ is a dimensionless wavenumber, $t$ is time, $\omega$ is the radian frequency, and $i=\sqrt{-1}$. For a steel substrate of inner radius $a_{1}$ and outer radius $b_{1}$ (this is region s, with a substrate wall thickness $t_{s}=b_{1}-a_{1}$ ), and a pipe coating of inner radius $b_{1}$ and outer radius $b_{2}$ (this is region c, with a coating thickness $t_{c}=b_{2}-b_{1}$ ), the substitution of Eq. (3) into Eq. (2) yields the Helmholtz equation

$$
\frac{\sim \cdot{ }_{\mathrm{s}, \mathrm{c}}}{\partial r^{2}}+\frac{1}{r} \frac{\sim_{\mathrm{s}, \mathrm{c}}}{\partial r}-\frac{\cdot_{\mathrm{s}, \mathrm{c}}}{r^{2}}+\left[k_{\mathrm{s}, \mathrm{c}}^{2}-k_{\mathrm{s}}^{2} \lambda^{2}\right] v_{\mathrm{s}, \mathrm{c}}=0 .
$$

The appropriate radial boundary conditions on the inner and outer walls are

$$
\mathbf{1}_{r \theta_{\mathrm{s}}}=\mathrm{u} \text {, at } r=a_{1} \text {, and } \boldsymbol{1}_{r \theta_{\mathrm{c}}}=\mathrm{u} \text { at } r=b_{2} \text {, }
$$

where $T_{r \theta_{s, c}}$ is the shear stress in the $r, \theta$ plane, with

$$
\boldsymbol{I}_{r \theta_{\mathrm{s}, \mathrm{c}}}=\mu_{\mathrm{s}, \mathrm{c}}\left\lfloor\frac{\cdots,-}{\partial r}-\frac{\omega,}{r}\right\rfloor .
$$


At the interface between the pipe substrate and coating, continuity of shear stress and displacement is enforced, so that

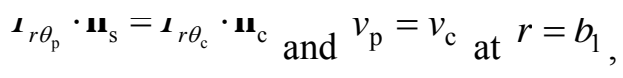

where $\mathbf{n}$ is the (outward) unit normal in each region. A standard finite element analysis is adopted here so that

$$
v(r)=\sum_{j=1} I \mathbf{v}_{j} v_{j}
$$

where $N_{j}$ is a global trial (or shape) function for the (transverse) one dimensional finite element mesh, $v_{j}$ is the value of $v(r)$ at node $j$, and $n$ is the number of nodes (or degrees of freedom) lying on the pipe cross section $a_{1} \leq r \leq b_{2}$. Expressing Eq. (8) in vector form yields

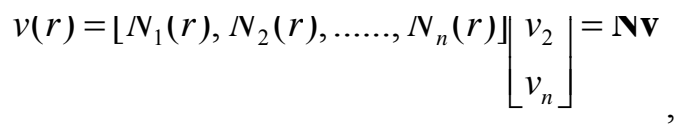

Following Kirby [6], the governing eigenequation for torsional modes may be written as

$$
\mathbf{A v}+\lambda^{2} \mathbf{B v}=\mathbf{0}
$$

where $\mathbf{v}=\left[\begin{array}{ll}\mathbf{v}_{\mathrm{s}} & \mathbf{v}_{\mathrm{c}}\end{array}\right]^{\mathrm{T}}$, so that

$$
\begin{aligned}
& \mathbf{A v}=\mathbf{A}_{\mathrm{s}} \mathbf{v}_{\mathrm{s}}+\beta \mathbf{A}_{\mathrm{c}} \mathbf{v}_{\mathrm{c}}, \\
& \mathbf{B v}=\mathbf{B}_{\mathrm{s}} \mathbf{v}_{\mathrm{s}}+\beta \mathbf{B}_{\mathrm{c}} \mathbf{v}_{\mathrm{c}}
\end{aligned}
$$

and

$$
\begin{gathered}
\mathbf{A}_{\mathrm{s}, \mathrm{c}}=\mathbf{K}_{1}^{\mathrm{s}, \mathrm{c}}-\mathbf{K}_{2}^{\mathrm{s}, \mathrm{c}}+\mathbf{M}_{2}^{\mathrm{s}, \mathrm{c}}-k_{\mathrm{s}, \mathrm{c}}^{2} \mathbf{M}_{1}^{\mathrm{s}, \mathrm{c}} \\
\mathbf{B}_{\mathrm{s}, \mathrm{c}}=k_{\mathrm{s}}^{2} \mathbf{M}_{1}^{\mathrm{s}, \mathrm{c}}
\end{gathered}
$$


with $\beta=\rho_{c} \mu_{\mathrm{c}} / \rho_{\mathrm{s}} \mu_{\mathrm{s}}$. In addition,

$$
\begin{aligned}
& \mathbf{\mathbf { n }}_{1}=\int_{a_{1}} \overline{\partial r} \overline{\partial r} \mathbf{u} \mathbf{u}+\left.\overline{a_{1}}\right|_{r=a_{1}}-\left.\overline{b_{1}}\right|_{r=b_{1}}, \\
& \mathbf{n}_{1}=\int_{b_{1}} \overline{\partial r} \overline{\partial r} r \mathbf{u}+\left.\overline{b_{1}}\right|_{r=b_{1}}-\left.\overline{b_{2}}\right|_{r=b_{2}}, \\
& \mathbf{n}_{2}=\int_{a_{1}} \frac{}{r} \frac{}{\partial r} r a r, \mathbf{n}_{2}=\int_{b_{1}} \frac{}{r} \frac{\bar{\partial}}{\partial r} r a r, \\
& \mathbf{I} \mathbf{M}_{1}^{-}=\int_{a_{1}} \mathbf{N}^{-} \mathbf{N} r a r \quad \mathbf{M} \mathbf{M}_{1}^{-}=\int_{b_{1}} \mathbf{N}^{-} \mathbf{N} r a r \\
& \mathbf{l} \mathbf{v}_{2}=\int_{a_{1}} \frac{}{r^{2}} r a r, \quad \mathbf{v} \mathbf{I}_{2}=\int_{b_{1}} \frac{}{r^{2}} r a r .
\end{aligned}
$$

Equation (10) is solved using a standard eigenvalue solver (in this case the eig function in MATLAB). This routine delivers $n$ unique (complex) eigenmodes and here the imaginary component is sorted in ascending order. The analysis in this section delivers a version of the SAFE method but for torsional modes only. However, the real power of the SAFE method lies in the use of the computed eigensolutions to study finite axial area discontinuities, or defects, with a computational cost that does not depend on the length of the (normally) uniform pipe sections either side of the defect. The following sections describe two different approaches for utilising the SAFE method: (i) mode matching for a uniform defect, and (ii) a hybrid numerical method for non-uniform defects.

\subsection{Mode Matching}

Mode matching is a straightforward technique for studying scattering from uniform discontinuities and this approach is used here to analyse an axisymmetric uniform notch of length 
$L_{N}$ and radius $a_{2}$, located within a coated pipe section (see Fig. 1). In Fig. 1, region 1 denotes an uncoated region within which the incident wave is generated, as this best replicates actual practice; the uniform notch is then located within a (semi-infinite) section of coated pipe. The displacement in region $q$ (where $q=1,2,3$, or 4 ) of the pipe is written as an infinite sum over the pipe eigenmodes, to give

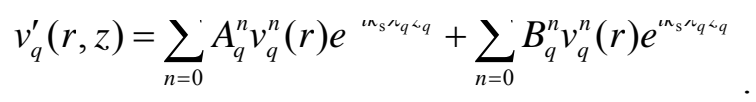

Here, ${ }^{A_{q}}$ and $B_{q}$ are the modal amplitudes in region $q$, and $z_{1}=z_{2}$. Mode matching proceeds by enforcing axial continuity conditions over planes A, B and C in Fig. 1. The appropriate continuity conditions over each plane are (i) continuity of displacement, and (ii) continuity of the shear stress $T_{\theta Z}^{\prime}$, where $T_{\theta Z}^{\prime}=\mu \partial v^{\prime} / \partial z$. In addition, the boundary condition $T_{\theta Z}^{\prime}=0$ is enforced over $b_{1}<r \leq b_{2}$ on plane A, and over $a_{2}<r \leq b_{2}$ on planes B and C. The continuity conditions yield

$$
\begin{aligned}
& v_{1}^{\prime}(r, 0)=v_{2}^{\prime}(r, 0), \quad \text { over } \quad a_{1} \leq r \leq b_{1} \text {; } \\
& \boldsymbol{I}_{\theta Z_{1}}(r, \mathrm{U})=\boldsymbol{\Lambda}_{\theta Z_{2}}(r, \mathrm{U}), \quad \text { over } \quad a_{1} \leq r \leq b_{2} ; \\
& v_{2}\left(r, L_{C}\right)=v_{3}(r, \mathrm{U}), \quad \text { over } \quad a_{1} \leq r \leq a_{2} \\
& \mathbf{1}_{\theta Z_{2}}\left(r, L_{C}\right)=\mathbf{1}_{\theta Z_{3}}(r, \mathrm{U}), \quad \text { over } \quad a_{1} \leq r \leq b_{2} \\
& v_{3}^{\prime}\left(r, L_{N}\right)=v_{4}(r, \mathrm{U}), \quad \text { over } \quad a_{1} \leq r \leq a_{2} \\
& \mathbf{1}_{\theta Z_{3}}\left(r, L_{N}\right)=\mathbf{1}_{\theta Z_{4}}(r, \mathrm{U}), \quad \text { over } \quad a_{1} \leq r \leq b_{2} .
\end{aligned}
$$

Here, the (normalised) shear stress is given as

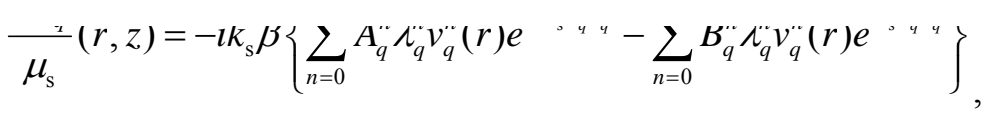


where $\widetilde{\beta}=1$ in an uncoated region, and $\widetilde{\beta}=\beta$ in a coated region. Following Kirby [16], the axial boundary conditions are enforced by choosing an appropriate weighting function and then integrating over the relevant cross section. For torsional modes, the most straightforward weighting function to choose is the respective pipe eigenfunction and, after application to each matching condition, this gives

$$
\begin{aligned}
& \int_{a_{1}} v_{1}^{\cdots}(r) v_{1}(r, \mathrm{u}) r a r=\int_{a_{1}} v_{1}^{\cdots}(r) v_{2}(r, \mathrm{u}) r a r \\
& \int_{a_{1}} v_{2}^{\cdots}(r) I_{\theta Z_{1}}(r, \mathrm{U}) r a r=\int_{a_{1}} v_{2}^{\cdots}(r) I_{\theta Z_{2}}(r, \mathrm{u}) r a r \\
& \int_{a_{1}} v_{3}(r) v_{2}\left(r, L_{\mathrm{c}}\right) r u r=\int_{a_{1}} v_{3}(r) v_{3}(r, \mathrm{v}) r \mathrm{r} r \\
& \int_{a_{1}} v_{2}(r) 1_{\theta Z_{2}}\left(r, L_{\mathrm{c}}\right) r u r=\int_{a_{1}} V_{2}(r) \mathbf{1}_{\theta Z_{3}}(r, u) r u r \\
& \int_{a_{1}} v_{3}^{\prime \prime}(r) v_{3}^{\prime}\left(r, L_{N}\right) r a r=\int_{a_{1}} v_{3}^{\prime \prime}(r) v_{4}(r, \mathrm{u}) r a r \\
& \int_{a_{1}} v_{4}^{\cdots}(r) I_{\theta Z_{3}}\left(r, L_{N}\right) r a r=\int_{a_{1}} v_{4}^{\cdots}(r) I_{\theta Z_{4}}(r, u) r a r
\end{aligned}
$$

Here, the weighting functions are summed over $m$, from 0 to $n_{q}$, where the summation has been omitted for clarity. Substitution of Eqs. (20) and (27) into the matching conditions yields six coupled equations. Solution of these equations requires a forcing term to be identified and the application of an axial boundary condition at $Z_{4}=\infty$. Here, the pipe is assumed to be driven by a plane wave (the "fundamental" torsional mode), that is $A_{1}^{0}=1$, and $A_{1}^{n}=0$ for $n>0$; in region 4 it is assumed that no reflections exist, so that $B_{4}^{n}=0$ for $n \geq 0$. Substituting these axial boundary conditions into the relevant matching conditions, and re-arranging so that the forcing terms lie on the right hand side, yields 


$$
\begin{aligned}
& B_{1}^{n} I_{11}^{m n}-A_{2}^{n} I_{12}^{m n}-\widetilde{B}_{2}^{n} I_{12}^{m n} e^{-i k_{\mathrm{s}} \lambda_{2}^{n} L_{\mathrm{C}}}=-A_{1}^{0} I_{11}^{m 0} \\
& \lambda_{1}^{n} B_{1}^{n} I_{21}^{m n}+\lambda_{2}^{n} A_{2}^{n} I_{22}^{m n}-\lambda_{2}^{n} \widetilde{B}_{2}^{n} I_{22}^{m n} e^{-i k_{s} \lambda_{2}^{n} L_{C}}=\lambda_{1}^{0} A_{1}^{0} I_{21}^{m 0}, \\
& A_{2}^{n} I_{32}^{m n} e^{-l \kappa_{\mathrm{s}} \varkappa_{2} L_{\mathrm{C}}}+B_{2}^{n} I_{32}^{m n}-A_{3}^{n} I_{33}^{m n}-B_{3}^{n} I_{33}^{m n} e^{-l \kappa_{\mathrm{s}} \Lambda_{3} L_{\mathrm{N}}}=0, \\
& \lambda_{2}^{n} A_{2}^{n} I_{22}^{m n} e^{-l \kappa_{\mathrm{s}} \wedge_{2} L}-\lambda_{2}^{n} B_{2}^{n} I_{22}^{m n}-\lambda_{3}^{n} A_{3}^{n} I_{23}^{m n}+\lambda_{3}^{n} B_{3}^{n} I_{23}^{m n} e^{-l \kappa_{\mathrm{s}} \bigwedge_{3} L_{\mathrm{N}}}=0, \\
& A_{3}^{n} I_{33}^{m n} e^{-l \kappa_{s} \iota_{3} L_{N}}+B_{3}^{n} I_{33}^{m n}-A_{4}^{n} I_{32}^{m n}=0, \\
& \lambda_{3}^{n} A_{3}^{n} I_{23}^{m n} e^{-l k_{s} \lambda_{3} L}-\lambda_{3}^{n} B_{3}^{n} I_{23}^{m n}-\lambda_{2}^{n} A_{4}^{n} I_{22}^{m n}=0
\end{aligned}
$$

Here, it is assumed that regions 2 and 4 are identical, $\widetilde{B}_{2}^{n}=B_{2}^{n} e^{-i k_{\mathrm{s}} \lambda_{2}^{n} L_{C}}$ and $\widetilde{B}_{3}^{n}=B_{3}^{n} e^{-i k_{\mathrm{s}} \lambda_{3}^{n} L_{N}}$. The integrals are given as

$$
\begin{aligned}
& \boldsymbol{I}_{11}=\int_{a_{1}} v_{1}^{\cdots}(r) v_{1}^{\cdots}(r) r a r \\
& I_{12}=\int_{a_{1}} v_{1}^{\cdots}(r) v_{2}^{*}(r) r a r \\
& \boldsymbol{I}_{21} \ldots \int_{a_{1}} v_{2}^{\cdots}(r) v_{1}(r) r a r \\
& I_{22}=\int_{a_{1}} v_{2}(r) v_{2}^{*}(r) r a r+\beta \int_{b_{1}} v_{2}^{\cdots}(r) v_{2}(r) r a r \\
& I_{33} \ldots \int_{a_{1}} v_{3}^{\cdots}(r) v_{3}^{\prime \prime}(r) r a r \\
& I_{32} \cdots=\int_{a_{1}} v_{3} \cdots(r) v_{2}^{*}(r) r a r \\
& I_{23}^{\cdots \cdots}=\int_{a_{1}} v_{2}^{\cdots}(r) v_{3}^{*}(r) r a r
\end{aligned}
$$


The integrals in Eqs. (40) - (46) are carried out numerically after truncating each summation at $n_{q}$. Equations (34) - (39) are then solved simultaneously for the unknown modal amplitudes.

\subsection{Hybrid numerical method}

The mode matching method described in the previous section is applicable only to uniform area discontinuities. For non-uniform discontinuities it is convenient to return to a full finite element discretisation of the defect in order to provide the flexibility to examine arbitrary (but axisymmetric) geometries. Accordingly, a hybrid method is adopted here, which follows the method described by Kirby [17] for acoustic waveguides. The advantage of this method is that it only requires a finite element discretisation of the non uniform defect and so retains the modal expansions within the uniform pipe sections. In general, this method may be applied to any three dimensional geometry; however, it is sensible first to investigate the method's effectiveness for simpler problems before moving on to examine fully three dimensional discontinuities. Accordingly, the hybrid method is applied here to an axisymmetric detect of arbitrary shape, thus restricting the problem to two dimensions and allowing the use of the one dimensional eigensolution discussed previously. The hybrid method relies on matching a modal representation of the sound field before and after the defect onto a full finite element discretisation of the sound field surrounding the defect, see for example the tapered defect in Fig. 2. Thus, in regions 1, 2 and 4 the modal expansions in Eqs. (20) and (27) still apply, whereas in region 3 the finite element method is used to solve Eq. (2) directly. Thus for region 3 a standard finite element discretisation is used, so that

$$
V_{3}^{\prime}(r, z)=\sum_{j=1} M_{j}(r, z) V_{3_{j}}
$$

where $M_{j}$ is a global trial (or shape) function for the (two dimensional) finite element mesh. Following Eq. (9), $v_{3}^{\prime}(r, z)=\mathbf{M} \mathbf{v}_{3}$, and application of a weak formulation to Eq. (4) yields, 


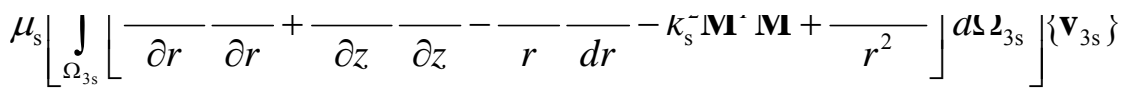

$$
\begin{aligned}
& =\mu_{\mathrm{s}} \int_{\Gamma_{3 \mathrm{~s}}} \mathbf{M}^{T} \frac{\partial v_{3 \mathrm{~s}}^{\prime}}{\partial \mathbf{n}_{3 \mathrm{~s}}} d \Gamma_{3 \mathrm{~s}}-\mu_{\mathrm{s}} \int_{\Gamma_{\mathrm{X}}} \mathbf{M}^{T} \frac{\partial v_{3 \mathrm{~s}}^{\prime}}{\partial \mathrm{z}} d \Gamma_{\mathrm{X}}+\mu_{\mathrm{s}} \int_{\Gamma_{\mathrm{Y}}} \mathbf{M}^{T} \frac{\partial v_{3 \mathrm{~s}}^{\prime}}{\partial \mathrm{z}} d \Gamma_{\mathrm{Y}} \text {. }
\end{aligned}
$$

for the pipe substrate, and

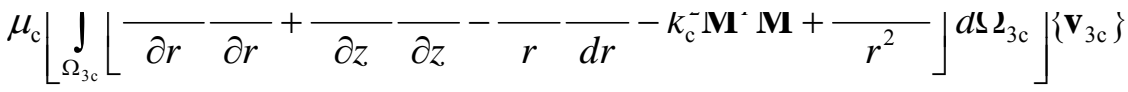

$$
\begin{aligned}
& =\mu_{c} \int_{\Gamma_{3 c}} \mathbf{M}^{T} \frac{\partial v_{3 c}^{\prime}}{\partial \mathbf{n}_{3 c}} d \Gamma_{3 c}-\mu_{c} \int_{\Gamma_{\mathrm{X}}} \mathbf{M}^{T} \frac{\partial v_{3 c}^{\prime}}{\partial z} d \Gamma_{\mathrm{X}}+\mu_{c} \int_{\Gamma_{\mathrm{Y}}} \mathbf{M}^{T} \frac{\partial v_{3 \mathrm{c}}^{\prime}}{\partial \mathrm{z}} d \Gamma_{\mathrm{Y}}
\end{aligned}
$$

for the coated region. Here, ${ }^{\mathbf{n}_{3 \mathrm{~s}, \mathrm{c}}}$ is a unit normal pointing outwards from surface ${ }^{\Gamma_{\mathrm{ss}, \mathrm{c}}} ; \Gamma_{\mathrm{X}}$ and $\Gamma_{\mathrm{Y}}$ denote the surface of $\Omega_{3 \mathrm{s,c}, \mathrm{c}}$ that lies on planes $\mathrm{X}$ and Y, respectively, and $\Gamma_{3 \mathrm{s,c}}$ is the surface of $\Omega_{3 \mathrm{~s}, \mathrm{c}}$ that does not lie on $\Gamma_{\mathrm{X}}$ and $\Gamma_{\mathrm{Y}}$ (see Fig. 2). In addition $\mathbf{v}_{3}=\left[\begin{array}{ll}\mathbf{v}_{3 \mathrm{~s}} & \mathbf{v}_{3 \mathrm{c}}\end{array}\right]^{\mathrm{T}}$ where $\mathbf{v}_{3 \mathrm{~s}}$ and $\mathbf{v}_{3 \mathrm{c}}$ are the displacements within the pipe and coating that lie in region 3, respectively.

The analysis proceeds by enforcing appropriate boundary conditions over the surface of $\Omega_{3 \mathrm{~s}, \mathrm{c}}$. Equation (7) applies over the interface between the coated $\left(\Gamma_{3 \mathrm{c}}\right)$ and uncoated $\left({ }^{\Gamma_{3 \mathrm{~s}}}\right)$ regions; and for the outer surface of $\Omega_{3 \mathrm{~s}, \mathrm{c}}\left({ }^{\Gamma_{3 \mathrm{~s}, \mathrm{c}}}\right)$ that does not lie on this interface, $T_{r \theta}^{\prime}=0$, which gives

$$
\overline{\partial \mathbf{n}_{3 s, \mathrm{c}}}=\frac{}{r} \cdot \mathbf{n}_{3 \mathrm{~s}, \mathrm{c}} .
$$

Application of these boundary conditions to Eqs (48) and (49) allows the two equations to be combined to give 


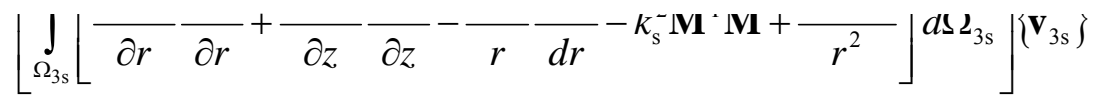

$$
\begin{aligned}
& +\beta\left[\int_{\Omega_{3 \mathrm{c}}}\left[\frac{\partial \mathbf{M}^{\mathrm{T}}}{\partial r} \frac{\partial \mathbf{M}}{\partial r}+\frac{\partial \mathbf{M}^{\mathrm{T}}}{\partial z} \frac{\partial \mathbf{M}}{\partial z}-\frac{\mathbf{M}^{\mathrm{T}}}{r} \frac{d \mathbf{M}}{d r}-k_{\mathrm{c}}^{2} \mathbf{M}^{\mathrm{T}} \mathbf{M}+\frac{\mathbf{M}^{\mathrm{T}} \mathbf{M}}{r^{2}}\right] d \Omega_{3 \mathrm{c}}\right]\left\{\mathbf{v}_{3 \mathrm{c}}\right\} \\
& +\left[\int_{\Gamma_{3 \mathrm{~s}}} \frac{\mathbf{M}^{\mathrm{T}} \mathbf{M}}{r} \cdot \mathbf{n}_{3 \mathrm{~s}} d \Gamma_{3 \mathrm{~s}}\right]\left\{\mathbf{v}_{3 \mathrm{~s}}\right\}+\beta\left[\int_{\Gamma_{3 \mathrm{c}}} \frac{\mathbf{M}^{\mathrm{T}} \mathbf{M}}{r} \cdot \mathbf{n}_{3 \mathrm{c}} d \Gamma_{\mathrm{c}}\right]\left\{\mathbf{v}_{3 \mathrm{c}}\right\} \\
& +\int_{\Gamma_{\mathrm{X}}}\left[\mathbf{M}^{\mathrm{T}} \frac{\partial v_{3 \mathrm{~s}}^{\prime}}{\partial z}+\beta \mathbf{M}^{\mathrm{T}} \frac{\partial v_{3 \mathrm{c}}^{\prime}}{\partial z}\right] d \Gamma_{\mathrm{X}}-\int_{\Gamma_{\mathrm{Y}}}\left[\mathbf{M}^{\mathrm{T}} \frac{\partial v_{3 \mathrm{~s}}^{\prime}}{\partial z}+\beta \mathbf{M}^{\mathrm{T}} \frac{\partial v_{3 \mathrm{c}}^{\prime}}{\partial z}\right] d \Gamma_{\mathrm{Y}}=0
\end{aligned}
$$

The hybrid method now requires the finite element discretisation of region 3 to be joined to regions 2 and 4 and this is accomplished here using mode matching. Accordingly, over $\Gamma_{\mathrm{X}}$ continuity of the (normalised) shear stress $T_{\theta Z}^{\prime}$ yields

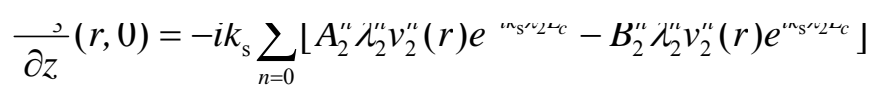

and over $\Gamma_{\mathrm{Y}}$

$$
\frac{\partial}{\partial z}\left(r, L_{3}\right)=-i k_{\mathrm{s}} \sum_{n=0} A_{4}^{\prime \prime} \lambda_{2}^{\prime \prime} v_{2}^{\prime \prime}(r)
$$

Here, it is assumed that there are no reflections in region 4 , so that $B_{4}^{n}=0, n=0 \ldots \infty$, and region 2 is identical to region 4 . Note that the hybrid method automatically removes the need to specify a perfectly matched layer (PML), which reduces computational expenditure, as well as enforcing the semi-infinite boundary condition exactly. Substitution of Eqs. (52) and (53) into Eq. (51) allows the shear stress matching conditions to be enforced, which after some re-arranging yields

$$
\mathbf{G} \mathbf{v}_{3}-\mathbf{D Q}^{1} A_{2}^{\prime \prime}+\mathbf{Q}^{1} B_{2}^{\prime \prime}+\mathbf{R}^{1} A_{4}^{\prime \prime}=\mathbf{0}
$$

where $\mathbf{D}$ is a diagonal matrix, with each diagonal element given by $e^{-i k_{\mathrm{s}} \lambda_{n} L_{\mathrm{c}}}\left(n=0,1, \ldots, n_{2}\right)$, and $\mathbf{D} \widetilde{B}_{2}^{n}=B_{2}^{n}$. In addition 


$$
\begin{aligned}
& \mathbf{G}=\int_{\Omega_{3 \mathrm{~s}}}\left[\frac{}{\partial r} \frac{}{\partial r}+\frac{}{\partial z} \frac{}{\partial z}-\frac{}{r} \frac{}{d r}-\boldsymbol{k}_{\mathrm{s}}^{-} \mathbf{M} \mathbf{M} \mathbf{M}+\frac{r^{2}}{a \iota_{3 \mathrm{~s}}}+\int_{\Gamma_{3 \mathrm{~s}}} \frac{r}{r} \cdot \mathbf{n}_{3 \mathrm{~s}} a \mathbf{l}_{3 \mathrm{~s}}\right. \\
& +\beta \int_{\Omega_{3 \mathrm{c}}}\left[\frac{\partial \mathbf{M}^{\mathrm{T}}}{\partial r} \frac{\partial \mathbf{M}}{\partial r}+\frac{\partial \mathbf{M}^{\mathrm{T}}}{\partial z} \frac{\partial \mathbf{M}}{\partial z}-\frac{\mathbf{M}^{\mathrm{T}}}{r} \frac{d \mathbf{M}}{d r}-k_{\mathrm{c}}^{2} \mathbf{M}^{T} \mathbf{M}+\frac{\mathbf{M}^{\mathrm{T}} \mathbf{M}}{r^{2}}\right] d \Omega_{3 \mathrm{c}}+\beta \int_{\Gamma_{3 \mathrm{c}}} \frac{\mathbf{M}^{\mathrm{T}} \mathbf{M}}{r} \cdot \mathbf{n}_{3 \mathrm{c}} d \Gamma_{3 \mathrm{c}}
\end{aligned}
$$

and

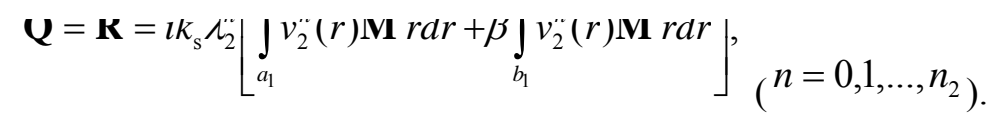

Continuity of displacement is matched separately, so that

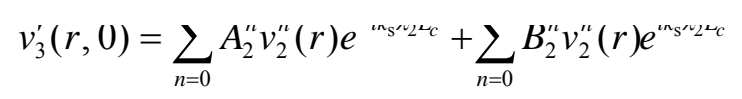

on $\Gamma_{\mathrm{x}}$, and

$$
v_{3}^{\prime}\left(r, L_{N}\right)=\sum_{n=0} A_{4}^{\prime \prime} v_{2}^{\prime \prime}(r)
$$

on $\Gamma_{\mathrm{Y}}$. Mode matching is used to enforce these matching conditions and this is implemented by multiplying Eqs. (57) and (58) by an appropriate weighting function. Following the method described by Kirby [17], a weighting function of $-i k_{\mathrm{s}} \lambda_{2}^{m} v_{2}^{m}$ for $\Gamma_{\mathrm{X}}$ is chosen, which, when multiplied by Eq. (57) yields,

$$
-\mathbf{D P} A_{2}^{\prime \prime}-\mathbf{P} B_{2}^{\prime \prime}+\mathbf{Q} \mathbf{v}_{3 X}=0
$$

where,

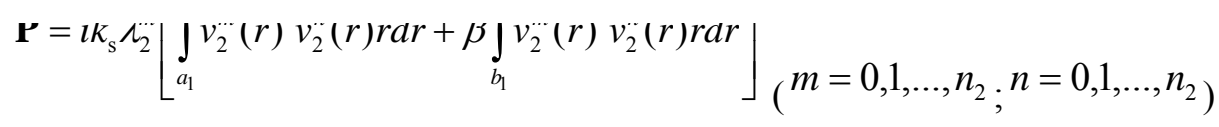

Similarly, for $\Gamma_{\mathrm{Y}}$

$$
-\mathbf{P} A_{4}^{\prime \prime}+\mathbf{K} \mathbf{v}_{3 \mathrm{Y}}=\mathbf{U}
$$


Here, vectors $\mathbf{v}_{3 \mathrm{X}}$ and $\mathbf{v}_{3 \mathrm{Y}}$ hold values of the finite element solution in region 3 at the nodal locations on the surfaces $\Gamma_{\mathrm{X}}$ and $\Gamma_{\mathrm{Y}}$, respectively. Finally, mode matching is used to accommodate the discontinuity between regions 1 and 2 using the same method as that described in the previous section, see Eq. (34) and (35). To combine Eqs. (54), (59), (61), (34) and (35), matrix $\mathbf{G}$ is first decomposed in order to separate those elements that lie on planes B and C, which allows the final system of equations to be written as

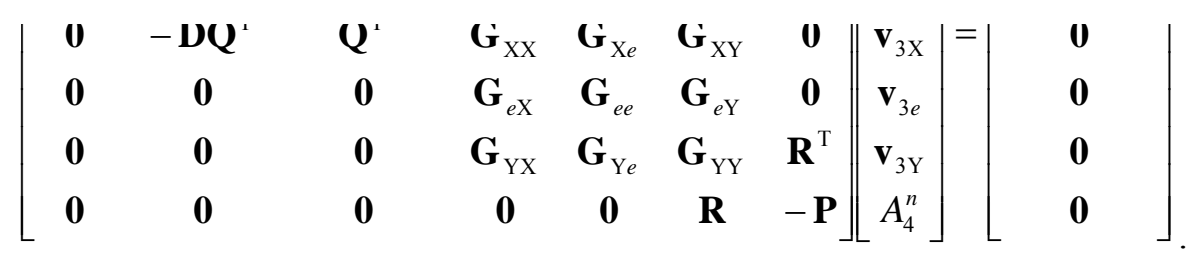

Here, the matrix $\mathbf{G}_{m n}$ has order $p_{m} \times p_{n}$, where $p_{2}$ and $p_{4}$ denote the number of nodes on $\Gamma_{\mathrm{X}}$ and $\Gamma_{\mathrm{Y}}$, respectively (where, $n_{2} \leq p_{2}$, and $n_{4} \leq p_{4}$ ); $p_{3}$ is the number of nodes in region 3 , and $S_{e}$ is the number of nodes that lie in region 3 , but do not lie on $\Gamma_{\mathrm{X}}$ and $\Gamma_{\mathrm{Y}}$ (so that $\left.p_{e}=p_{3}-p_{2}-p_{4}\right)$. The values for displacement at those nodes in region 3 that do not lie on $\Gamma_{\mathrm{X}}$ and $\Gamma_{\mathrm{Y}}$, are held in matrix $\mathbf{v}_{3 e}$. Equation (62) consists of $p_{T}\left(=n_{1}+2 n_{2}+p_{3}+n_{4}\right)$ simultaneous equations, which are solved to give the unknown modal amplitudes and displacements. 


\section{EXPERIMENT}

Validation of the model reported in the previous section is accomplished here by comparing predictions to data available in the literature and data obtained experimentally. The use of experimental data is particularly important for analysing the effects of a coating since very little measured data is present in the literature for coated pipes. Accordingly, a set of experiments are undertaken here that seek to measure the reflection coefficient of uniform defects located in coated and uncoated steel pipes. The steel pipe analysed here is a 3 inch Schedule 40 pipe with

$a_{1}=39 \mathrm{~mm}$ and $b_{1}=44.65 \mathrm{~mm}$. The test pipe has an overall length of $6 \mathrm{~m}$ and a defect is machined into the pipe at a distance of $0.8 \mathrm{~m}$ from the far end of the pipe; the geometry of each defect is reported in the next section.

In order to excite the fundamental $T(0,1)$ torsional mode a commercial Teletest device [31] is used, with 16 transducers equally spaced around the pipe circumference. These transducers are placed in region 1 of the pipe (see Fig. 1) at a distance of $5.1 \mathrm{~m}$ from the defect. For all tests, an incident pulse consisting of 10 cycles of a Hann modulated tone burst signal is used, with a centre excitation frequency that may be varied from $20 \mathrm{kHz}$ to $120 \mathrm{kHz}$. The reflected pulse is captured by a separate Teletest unit with four transducers equally spaced around the pipe in region 1; this unit was placed $1 \mathrm{~m}$ from the defect for an uncoated pipe, and $1 \mathrm{~m}$ from the edge of the coating for a coated pipe. The captured pulse was averaged 32 times in order to reduce levels of incoherent noise. The reflection coefficient is then calculated in the frequency domain after the application of a rectangular window to the (time domain) pulse and the use of an FFT. A large number of tests were undertaken over a small frequency range to enable the full frequency range to be covered, using a frequency step of approximately $125 \mathrm{~Hz}$. Up to ten repeat tests were conducted for each frequency range and an average taken. The results obtained from these 
experiments are reported in the following section, although it is noted that in order to provide clarity when viewing this data a frequency bandwidth of $1.25 \mathrm{kHz}$ is used and intermediate data discarded.

For a coated pipe, an identical set of experiments were carried out, but with a coating of length of $L_{c}=0.995 \mathrm{~m}$ upstream of the defect, and with the $0.8 \mathrm{~m}$ length of pipe on the other side of the defect also coated. The coating material used here was bitumen with a thickness of $1.5 \mathrm{~mm}$. The velocity of a bulk shear wave within a viscoelastic material may be written as [2]

$$
C_{c}=1 /\left\lfloor\frac{\overline{C_{c}}}{\widetilde{C}_{c}}+1 \dot{\bar{\omega}}\right\rfloor,
$$

where $\widetilde{C}_{c}$ corresponds to the phase velocity of a bulk shear wave travelling in the viscoelastic coating, and $\alpha_{c}$ corresponds to the attenuation of the shear wave (in Nepers $/ \mathrm{m}$ ). It is convenient here to write $\widetilde{\alpha}_{c}=\alpha_{c} / \omega$, and Barshinger and Rose [2] measured values of $\widetilde{\alpha}_{c}=0.24 \times 10^{-3} \mathrm{~s} / \mathrm{m}$ and $\widetilde{c}_{c}=770 \mathrm{~m} / \mathrm{s}$ for a bitumen based "Bitumastic 50" coating; alternative values for bitumen are also reported by Hua and Rose [14], as well as Simonetti and Cawley [32], who measured values of $\widetilde{\alpha}_{c}=0.122 \times 10^{-3} \mathrm{~s} / \mathrm{m}$ and $\widetilde{c}_{c}=750 \mathrm{~m} / \mathrm{s}$ for a solid bitumen rod. These values are reviewed in the following section when comparing prediction and measurement, although the non-dimensional parameter $\beta$, defined before Eq. (15), also requires data for the density of bitumen and here values of $\rho_{c}=1500 \mathrm{~kg} / \mathrm{m}^{3}$ and $\rho_{c}=1750 \mathrm{~kg} / \mathrm{m}^{3}$ are specified by Barshinger and Rose, and Simonetti and Cawley, respectively. 


\section{RESULTS AND DISCUSSION}

It is sensible first to validate the numerical model for an uncoated pipe since this allows for comparison against simple analytic models as well as experimental data available in the literature. Accordingly, an uncoated pipe is examined first for uniform and non-uniform defects.

\subsection{Uncoated pipe}

If one restricts wave propagation to the fundamental torsional mode only (otherwise called here a plane wave) then a simple analytic relationship is available for calculating the reflection coefficient of a uniform defect. For a pipe of inner radius $a_{1}$ and outer radius $b_{1}$, and uniform defect of outer radius $a_{2}$, the plane wave reflection coefficient $\Lambda_{\mathrm{pw}}$ is given as

$$
\Lambda_{\mathrm{pw}}=\frac{-}{\left[\sigma^{2}+1\right]-2 i \sigma \cot \left(k_{\mathrm{s}} L_{N}\right)}
$$

where $\sigma=\left(a_{2}^{4}-a_{1}^{4}\right) /\left(b_{1}^{4}-a_{1}^{4}\right)$. Note that this expression is analogous to that used for expansion chambers in acoustic waveguides [33] and is derived in an identical way. Here, a comparison with the numerical models reported in Section 2 may readily be achieved by setting $\beta=0$ in the relevant equations. Accordingly, in Fig. 3 predictions obtained using the mode matching method of Section 2.1 are compared against the analytic expression given by Eq. (64) for a uniform defect with $a_{2}=41.85 \mathrm{~mm}$ (a $50 \%$ area reduction) and $L_{N}=15 \mathrm{~mm}$. For a steel pipe, the torsional bulk wave velocity $c_{s}=3260 \mathrm{~m} / \mathrm{s}$. The mode matching predictions shown in Fig. 3 were obtained using only 6 quadratic finite elements, which is sufficient to deliver convergence to two decimal places for the reflection coefficient at a frequency of $150 \mathrm{kHz}$. This corresponds to a final system of equations delivering a matrix with an order of 40, and so delivers virtually instantaneous predictions at each frequency. In Fig. 3 it is evident that the analytic solution 
correlates well with the mode matching method at very low frequencies, which is to be expected; however, at higher frequencies the two solutions begin to diverge from one another and this occurs within a frequency range where higher order modes do not propagate. Here, a rise in the reflection coefficient is seen for the mode matching method, as well as a frequency shift. For example, in Fig. 3 the second peak shifts from a value of $\Lambda_{\mathrm{pw}}=0.662$ at a frequency of 48.5 $\mathrm{kHz}$, to a multi modal value of $\Lambda=0.695$ at a frequency of $145 \mathrm{kHz}$. This effect can also be seen in the study of expansion chambers in acoustic waveguides [34]. This behaviour at higher frequencies is very useful for illustrating the limitations of a plane wave (or single mode) based analysis: the difference between the two methods is caused by the relative influence of higher order evanescent modes in the prediction of reflection coefficient. These evanescent modes are crucial to the delivery of a converged solution for the axial shear stress matching conditions [Eqs. (22), (24) and (26)], especially at higher frequencies. Here, Eq. (64) omits all evanescent modes and so applies continuity of shear stress over the whole area/volume (analogous to continuity of acoustic volume velocity in an acoustic waveguide) rather than over individual "particles" in the waveguide. As the frequency increases, evanescent modes play more of a role in delivering the correct axial continuity conditions in the mode matching scheme, and so the two methods begin to depart from one another. This simple example illustrates the importance of higher order modes to mode matching schemes and this is one reason why numerical methods for solving the eigenproblem are preferable since for more complex problems many evanescent roots are normally required (see Kirby [16] for a discussion on this issue for acoustic waveguides). The mode matching predictions in Fig. 3 may readily be extended to higher frequencies, however this has been omitted from Fig. 3 in order to focus on validating the method against experimental data, and here it is evident that very good correlation is obtained between the measured and predicted reflection coefficient up to approximately $115 \mathrm{kHz}$. Moreover, the level of agreement between prediction and measurement is comparable to that seen in other studies [29]. 
A traditional check on the validity of predictions for non dissipative systems is a simple power balance and for the mode matching method the sound power balances to machine accuracy for all frequencies shown in Fig. 3. Note, however, that for modal based solutions balancing sound power does not always provide reliable evidence that the model has been implemented correctly (see Lawrie and Guled [35]). Nevertheless the data reported in Fig. 3 demonstrates that the mode matching method is capable of providing accurate predictions for very modest or, one may argue, negligible computational expenditure. The mode matching method also offers a straightforward way of validating the hybrid method developed in Section 2.2, and for the data shown in Fig. 3, agreement to two decimal places was obtained for the two methods using a value of $p_{T}=121$ in the hybrid method (using eight noded quadrilateral elements in region 3). Obviously, the use of the hybrid method to solve problems for uniform defects is not computationally efficient, however the size of the numerical problem is very small and solutions can be still obtained very quickly. Of course, as the defect size increases the hybrid method will become slower in view of the additional elements required to accurately capture the behaviour within the defect (whereas the solution time for the mode matching method is not a function of the length of the defect).

One further issue with the hybrid method is the location of planes B and C. This has been discussed in detail for more complicated "obstacles" in acoustic waveguides by Kirby [17] and, in general, it was concluded that one should not locate these planes close to areas in which modal scattering is pronounced. This is because a modal solution often requires a proportionally larger number of modes to accurately represent a complex sound field when compared to the use of a standard finite element discretisation. Therefore, there exists an optimum location for planes B and $\mathrm{C}$ in which the efficiency of the approach is maximised, so that the size of the finite element mesh is balanced against the number of modes required in the wave based solution. However, the problems studied here are sufficiently straightforward to allow planes B and C to be located very close to (but not coincident with) each area discontinuity. 
The purpose of the hybrid method is of course to study non uniform area discontinuities. Therefore, the reflection coefficients for two tapered defects are shown in Fig. 4. These defects have been chosen to replicate those studied by Carandente et al. [29] and here a value of $\gamma=30^{\circ}$ is chosen for the taper angle, with $a_{1}=32.6 \mathrm{~mm}$ and $b_{1}=38.1 \mathrm{~mm}$ (see Fig. 2). In Fig. 4 the reflection coefficient for $\gamma=90^{\circ}$ is also included (this is a uniform defect of the type seen in Fig. 3), as well as a "V" shaped defect with a taper angle of $\gamma=11^{\circ}$. Here, a "V" shaped defect is obtained by setting $L_{N}$ to be very small (a value of $0.001 \mathrm{~mm}$ was used). To facilitate comparison with Carandente et al. [29], the reflection coefficient in Fig. 4 is plotted against the nondimensional variable $100 L_{e} / \lambda^{\prime}$, where $L_{e}$ is the equivalent length of the defect and $\lambda^{\prime}$ is the wavelength of sound. For an uncoated pipe, $L_{e}=L_{N}+\left(b_{1}-a_{1}\right) \cot \gamma$. The results presented in Fig. 4 compare very well with those predictions reported by Carandente et al. [29] and this provides further validation of the hybrid and mode matching methods developed here. However, Carandente et al. used a fully three dimensional finite element discretisation of the entire pipe to generate their theoretical predictions and so it is clear that the current computational techniques will be significantly faster. Furthermore, the large reduction in degrees of freedom also allows predictions to be extended to higher frequencies with relative ease, and both methods in Section 2 are suitable for uniform pipes of arbitrary length, whereas a full FE discretisation will quickly become very expensive as the length of the pipe increases. In Fig. 4 it is also noticeable that the effect of the taper on the reflection coefficient is significant only at higher values of $L_{e} / \lambda^{\prime}$,

A further validation of the hybrid method may be obtained by comparing predictions against experimental measurements taken for a tapered defect. Accordingly, in Fig. 5 predictions are compared against measured data for the refection coefficient of a tapered defect in the same pipe as that used in Fig. 3 (see also discussions in Section 3); the angle of the taper is $\gamma=30^{\circ}$ and 
$L_{N}=15 \mathrm{~mm}$. In Fig. 5 generally good agreement is observed between prediction and experiment, although at lower and higher frequencies the agreement is not as good as that seen for the defect without a taper in Fig. 3. This difference is likely to be caused by experimental errors that may be attributable to an increase in coherent noise when a taper is present, although this observation remains to be supported by further investigation. The agreement between prediction and experiment seen in Fig. 5 is, however, generally comparable to that seen in the literature [29] and is accurate to within $10 \%$ below $90 \mathrm{kHz}$, which may be considered to be acceptable for this type of problem.

\subsection{Coated Pipe}

The presence of a viscoelastic coating is known to cause significant problems when identifying defects in pipes and so in Fig. 6 the reflection coefficient for a uniform defect is presented for a pipe coated with bitumen. The geometry of the pipe and the thickness of the coating is given in Section 3, and the uniform defect has a length $L_{N}=15 \mathrm{~mm}$. The theoretical predictions in Fig. 6 were obtained using the mode matching approach, with four quadratic elements for the steel substrate and two for the coated region, which delivers a converged solution for the reflection coefficient to two decimal places up to $140 \mathrm{kHz}$. The experimental data in Fig. 6 were obtained in exactly the same way as that seen for the uncoated pipe, although the transducers that detect the reflected pulse were moved $1 \mathrm{~m}$ away from the end of the bitumen coating.

In Fig. 6 three theoretical predictions are presented for a coated pipe. The first set of theoretical predictions is obtained using the material constants reported by Barshinger and Rose [2], so that $\tilde{\alpha}_{c}=0.24 \times 10^{-3} \mathrm{~s} / \mathrm{m}, \quad \widetilde{c}_{c}=750 \mathrm{~m} / \mathrm{s}$ and $\rho_{c}=1500 \mathrm{~kg} / \mathrm{m}^{3}$; the second set of predictions is obtained using data reported by Simonetti and Cawley [32], with $\tilde{\alpha}_{c}=0.122 \times 10^{-3} \mathrm{~s} / \mathrm{m}$, $\widetilde{c}_{c}=770 \mathrm{~m} / \mathrm{s}$ and $\rho_{c}=1750 \mathrm{~kg} / \mathrm{m}^{3}$. It is clear in Fig. 6 that when using data measured by 
Barshinger and Rose, or Simonetti and Cawley, there is a significant discrepancy between the predicted and measured reflection coefficients. Furthermore, it is noticeable that at higher frequencies the predicted amplitude of the reflection coefficients is significantly reduced and is seen almost to disappear beyond the first minima in the experimental data. In contrast the measured reflection coefficient in Fig. 6 is seen to follow a similar pattern to that seen for the uncoated pipe, albeit with lower values for the reflection coefficient. This behaviour is what one would expect to see if the bitumen coating was simply attenuating the axial sound propagation, with the influence of the coating on the scattering at the defect being relatively small. Clearly then the discrepancies seen in Fig. 6 serve to question the appropriateness of the theoretical model, or at least the appropriateness of the values reported in the literature when analysing the bitumen coating used in the current study. It is noticeable, moreover, that in the literature comparisons between prediction and experiment for coated pipes remains limited, and to date only small amounts of data have been measured for the attenuation of $\mathrm{T}(0,1)$, and even these are limited to frequencies above those studied here.

The third set of theoretical predictions in Fig. 6 is seen to agree much better with the experimental data. These predictions have been obtained after running parametric studies with different values of $\alpha_{c}$ and $\rho_{c}$, and choosing values that gives good agreement between prediction and experiment. Here, values of $\widetilde{\alpha}_{c}=3.9 \times 10^{-3} \mathrm{~s} / \mathrm{m}$ and $\rho_{c}=1200 \mathrm{~kg} / \mathrm{m}^{3}$ were identified, although a value of $\widetilde{C}_{c}=750 \mathrm{~m} / \mathrm{s}$ was retained. Thus, the parametric studies required a relatively modest change in the density of the coating, but a much larger change in the value of $\widetilde{\alpha}_{c}$ when compared to those reported in Refs. [2] and [32]. It was decided that ${ }^{{ }_{c}}$ should be the subject of larger variations in value because this is the most difficult parameter to measure independently and so the most uncertainty exists with this value; for example, a direct measurement of this parameter has yet to be realised over the frequency range shown in Fig. 6 for a thin sheet of bitumen typically found in industrial applications. Accordingly there is still a 
question mark over what constitutes an appropriate value for the constant $\widetilde{\alpha}_{c}$ for the particular type of material studied here and also how this value varies between, for example, differing types of bitumen. The results presented here suggest that a much higher value than that reported by Barshinger and Rose [2], and Simonetti and Cawley [32], is appropriate for the bitumen coating used in these experiments, although given the inherent uncertainty with this data it would probably be unwise to extrapolate this value to other types of bitumen before more experimental work has been undertaken. Thus, Fig. 6 demonstrates that it is possible to generate good agreement between prediction and theory for the coated pipe, but it appears necessary to do further work on the acoustic properties of coatings such as bitumen and, ideally, to develop a method that will independently measure $\widetilde{\alpha}_{c}$ for relatively thin sheets of bitumen that cannot be studied using methods such as the one described by Simonetti and Cawley [32].

The theoretical predictions in Fig. 6 are also seen to oscillate over the frequency range shown, although heavy damping is also seen in data at higher frequencies. These oscillations are caused by internal reflections within the coating material. The wavelength and amplitude of these oscillations is largely dictated by the length of the coating $\left({ }^{L_{c}}\right)$, and here a longer coating generally gives oscillations of shorter wavelength and higher amplitude. This effect can also be seen in later figures. It is, however, noticeable that the amplitude of the oscillations relative to the overall amplitude of the reflection coefficients shown in Fig. 6 is small and so one cannot expect to pick up these oscillations in the experimental data.

The theoretical analysis in Section 2 and the data reported in Fig. 6 assume that a finite length of coating is present before the defect so that the incident sound field may be generated within an uncoated section. This allows for a straightforward comparison between predictions obtained for coated and uncoated pipes since the same definition for reflection coefficient may be used. This does, however, assume that the discontinuity at plane A has a negligible influence on sound 
propagation. Accordingly, in Fig. 7 the reflection coefficient for this area change alone is investigated for different coating thicknesses using the data determined previously (with $\widetilde{\alpha}_{c}=3.9 \times 10^{-3} \mathrm{~s} / \mathrm{m}$ and $\rho_{c}=1200 \mathrm{~kg} / \mathrm{m}^{3}$ ). Here, the reflection coefficient for plane A on its own can readily be recovered by setting all the modal amplitudes in the mode matching model to zero, except for the modes scattered by plane A. In Fig. 7 the reflection coefficient is plotted against a non-dimensional variable $k_{s} t_{s}$, for different coating thicknesses of $t_{c} / t_{s}=0.25,0.5$, and 1 . Here, the reflection coefficient for the coated pipe studied in Fig. 6 is shown, although the data generated for other standard pipe sizes also closely fit the (nondimensional) curves shown in Fig. 7, and are omitted here only in the interests of clarity. Figure 7 indicates that in general the reflections from this area discontinuity are small when compared to the data presented in Fig. 6, although one can see an increase in values at lower frequencies. The data presented in Fig. 6 has a value of $t_{c} / t_{s}=0.3$ and so it appears justifiable to neglect the influence of the reflections from plane A when discussing the results in Fig. 6; however, it is evident that as the length of the coating increases, and the value of the reflection coefficient drops, the influence of plane A will become more significant, especially at higher values of $t_{c} / t_{s}$.

In order to validate the hybrid model for a coated pipe a defect similar to that used for the uncoated pipe is examined. Accordingly theoretical predictions for a coated pipe identical to that reported in Fig. 6 are presented in Fig. 8, but this time for a tapered defect with an angle of $\gamma=60^{\circ}$ and a defect length of $L_{N}=30 \mathrm{~mm}$. Thus, the previously optimised values of $\widetilde{\alpha}_{c}=3.9 \times 10^{-3} \mathrm{~s} / \mathrm{m}, \widetilde{c}_{c}=750 \mathrm{~m} / \mathrm{s}$ and $\rho_{c}=1200 \mathrm{~kg} / \mathrm{m}^{3}$ are used for the coating and predictions are compared here with experimental data obtained for a tapered defect machined into a coated pipe. It is evident in Fig. 8 that the level of agreement between prediction and measurement is similar to that seen in Fig. 6, although at higher frequencies the predictions tend to overestimate the reflection coefficient. It is also noticeable that at lower frequencies the measured reflection 
coefficient drops off rapidly and agreement between prediction and experiment is poor; however, it is likely that this is caused by experimental errors occurring at relatively low frequency, since in this frequency range the transducers are less efficient and are prone to higher errors. Nevertheless, the agreement between prediction and measurement over a wide frequency range is generally good in Figs. 6 and 8 and this suggests that the theoretical model is capable of capturing the physics of the problem.

In Fig. 9 the reflection coefficient for a "V" shaped defect $\left(\gamma=30^{\circ}\right)$ is investigated for a coated pipe by varying values of $t_{c} / t_{s}$ from 0.1 to 0.5 . Here, the length of the coating is increased to $2 \mathrm{~m}$, with $a_{1}=39 \mathrm{~mm}, a_{2}=41.85 \mathrm{~mm}, b_{1}=44.65 \mathrm{~mm}$ and $\widetilde{\alpha}_{c}=3.9 \times 10^{-3} \mathrm{~s} / \mathrm{m}$. The effect of increasing the length of the coating from $0.995 \mathrm{~m}$ to $2 \mathrm{~m}$ is immediately obvious in Fig. 9, and a significant lowering of the reflection coefficient is observed when compared to the uncoated pipe. This is consistent with axial attenuation in the coating playing a dominant role. Clearly the bitumen coating is predicted to have a significant effect on sound propagation and it is noticeable that the amplitude of the reflection coefficient is significantly reduced over the whole frequency range, even for relatively thin coatings. It is interesting also to note that the reflection coefficient for $t_{c} / t_{s}=0.5$ is higher than that for $t_{c} / t_{s}=0.25$ over a significant frequency range, which is not what one would expect; the reasons for this are not clear. The predictions in Fig. 9 do, however, clearly illustrate problems that are likely to be encountered when attempting to locate defects in coated pipes using the fundamental torsional mode: any increase in coating length is likely to further reduce the refection coefficient from the defect and make it increasingly difficult to locate the defect. 


\section{CONCLUSIONS}

The attenuation of guided elastic waves in coated pipes has been studied using two fast and efficient numerical methods. Both methods utilise the modal solutions delivered by the SAFE method and mode matching is used to study uniform defects, with a hybrid method used to analyse non-uniform defects. Good agreement between prediction and measurement for uncoated pipes is observed, and it is demonstrated that these numerical methods require very few degrees of freedom in order to study the scattering of torsional modes from axisymmetric defects in pipes.

The main aim of this article is to quantify reflection coefficients for axisymmetric defects in coated pipes and here it is seen that the use of data published in the literature for the bulk acoustic properties of bitumen does not provide good agreement between prediction and experiment. In order to rectify this, it was found to be necessary to change the value for $\alpha_{c}$, and a value up to 16 times larger than, for example, the data reported by Barshinger and Rose [2] was found to provide good agreement between prediction and measurement. Whilst this approach does demonstrate that it is possible to achieve good agreement between prediction and measurement such an approach is rather unsatisfactory. To provide more confidence in the values chosen here for $\alpha_{c}$ it is necessary in the future to develop an independent method for measuring $\alpha_{c}$ for the frequency range investigated, that is, the frequency range over which this technique is normally applied in the field and for the type of material normally encountered. This will enable conclusions to be drawn regarding the appropriateness of the values deduced here, and also the appropriateness of extrapolating data from higher frequencies [2]. Of course, further investigation is also necessary to see if the discrepancies are simply a result of the different type of bitumen used. It is, however, clear that the coating has a very pronounced effect on sound propagation and the results presented in Fig. 9 serve to question the feasibility of using the $\mathrm{T}(0,1)$ mode to locate defects in long lengths of coated pipelines. 
The theoretical methods reported here can readily be extended to other symmetric problems, such as longitudinal modes; they may also, in principle, be extended to the study of non-axisymmetric defects and the propagation of flexural waves. This is because the SAFE method is a general method for locating eigenmodes and it has been shown in the literature that this method will work equally well for a waveguide of arbitrary cross-section; moreover, the mode matching/hybrid methods can also in principle be extended to three dimensional problems. Therefore, given the potentially large computational savings observed in this current study it appears sensible in the future to investigate extending these techniques to more complicated scenarios for coated pipes. 


\section{REFERENCES}

1. C.W. Chan, P. Cawley, Lamb waves in highly attenuative plastic plates, Journal of the Acoustical Society of America 104 (1998) 874-881.

2. J.N. Barshinger, J.L.Rose, Guided wave propagation in an elastic hollow cylinder coated with a viscoelastic material, IEEE Transactions on Ultrasonics, Ferroelectrics, and Frequency Control 51 (2004) 1547-1556.

3. F. Simonetti, P. Cawley, On the nature of shear horizontal wave propagation in elastic plates coated with viscoelastic materials, Proceedings of the Royal Society London A 460 (2004), 2197-2221.

4. I. Bartoli, A. Marzani, F. Lanza di Scalea, E. Viola, Modeling wave propagation in damped waveguides of arbitrary cross-section, Journal of Sound and Vibration 295 (2006) 685-707.

5. R.J. Astley, A. Cummings, A finite element scheme for attenuation in ducts lined with porous material: comparison with experiment, Journal of Sound and Vibration 116 (1987) 239-263.

6. R. Kirby, Transmission loss predictions for dissipative silencers of arbitrary cross section in the presence of mean flow, Journal of the Acoustical Society of America 114 (2003) 200-209.

7. H. Taweel, S.B. Dong, M. Kazic, Wave reflection from the free end of a cylinder with an arbitrary cross section, International Journal of Solids and Structures, 37, (2000) 1701-1726.

8. T. Hayashi, K. Kawashima, Z. Sun, J.L. Rose, Analysis of flexural mode focusing by a semianalytical finite element method, Journal of the Acoustical Society of America 113 (2003) 1241-1248.

9. T. Hayashi, W-J. Song, J.L. Rose, Guided wave dispersion curves for a bar with an arbitrary cross-section, a rod and rail example, Ultrasonics 41 (2003) 175-183.

10. T. Hayashi, Guided wave animation using semi-analytical finite element method, $16^{\text {th }}$ World Conference in NDT, Montreal, Canada (2004). 
11. M. Castaings, M. Lowe, Finite element model for waves guided along solid systems of arbitrary section coupled to infinite solid media, Journal of the Acoustical Society of America 123 (2008) 696-708.

12. J. Mu, J.L.Rose, Guided wave propagation and mode differentiation in hollow cylinders with viscoelastic coatings, Journal of the Acoustical Society of America 124 (2008) 866-874.

13. A. Marzani, E. Viola, I. Bartoli, F. Lanza di Scalea, P. Rizzo, A semi-analytical finite element formulation for modeling stress wave propagation in axisymmetric damped waveguides, Journal of Sound and Vibration 318 (2008) 488-505.

14. J. Hua, J.L. Rose, Guided wave inspection penetration power in viscoelastic coated pipes, Insight 52 (2010) 195-200.

15. M.V. Predoi, M. Castaings, L. Moreau, Influence of material viscoelasticity on the scattering of guided waves by defects, Journal of the Acoustical Society of America 124 (2008) 28832894.

16. R. Kirby, A comparison between analytic and numerical methods for modelling automotive dissipative silencers with mean flow, Journal of Sound and Vibration 325 (2009) 565-582.

17. R. Kirby, Modeling sound propagation in acoustic waveguides using a hybrid numerical method, Journal of the Acoustical Society of America 124 (2008) 1930-1940.

18. H.E. Engan, Torsional wave scattering from a diameter step in a rod, Journal of the Acoustical Society of America 104 (1998) 2015-2024.

19. A. Demma, P. Cawley, M. Lowe, Scattering of the fundamental shear horizontal mode from steps and notches in plates, Journal of the Acoustical Society of America 113 (2003) 18801891.

20. J.J. Ditri, J.L. Rose, Excitation of guided elastic wave modes in hollow cylinders by applied surface tractions, Journal of Applied Physics 72 (1992) 2589-2597.

21. T. Vogt, M. Lowe, P. Cawley, The scattering of guided waves in partly embedded cylindrical structures, Journal of the Acoustical Society of America 113 (2003) 1258-1272. 
22. M.G. Imhof, Scattering of acoustic and elastic waves using hybrid multiple multipole expansions - Finite element technique, Journal of the Acoustical Society of America 100 (1996) 1325-1338.

23. W. Zhuang, A.H. Shah, S.K. Datta, Axisymmetric guided wave scattering by cracks in welded steel pipes, Journal of Pressure Vessel Technology 119 (1997) 401-406.

24. H. Bai, A.H. Shah, N. Popplewell, S.K. Datta, Scattering of guided waves by circumferential cracks in steel pipes, Journal of Applied Mechanics 68 (2001) 619-631.

25. W.J. Zhou, M.N. Ichchou, J.M. Mencik, Analysis of wave propagation in cylindrical pipes with local inhomogeneities, Journal of Sound and Vibration 319 (2009) 335-354.

26. P. Cawley, M.J.S. Lowe, F. Simonetti, C. Chevalier, A.G. Roosenbrand, The variation of the reflection coefficient of extensional guided waves in pipes from defects as a function of defect depth, axial extent, circumferential extent and frequency, Proceedings of the Institution of Mechanical Engineers, Part C 216 (2002) 1131-1143.

27. A. Demma, P. Cawley, M. Lowe, A.G. Roosenbrand, The reflection of the fundamental torsional mode from cracks and notches in pipes, Journal of the Acoustical Society of America 114 (2003) 611-625.

28. M. Ratassepp, S. Fletcher, M.J.S. Lowe, Scattering of the fundamental torsional mode at an axial crack in a pipe, Journal of the Acoustical Society of America 127 (2010) 730-740.

29. R. Carandente, J. Ma, P. Cawley, The scattering of the fundamental torsional mode from axisymmetric defects with varying depth profile in pipes, Journal of the Acoustical Society of America 127 (2010) 3440-3448.

30. J.D. Achenbach, Wave propagation in elastic solids, $8^{\text {th }}$ Ed. (Elsevier, Amsterdam, 1999).

31. http://www.plantintegrity.com/teletest/mini-tools.jsp

32. F. Simonetti, P. Cawley, Ultrasonic interferometry for the measurement of shear velocity and attenuation in viscoelastic solids, Journal of the Acoustical Society of America 115 (2004) 157-164. 
33. L.E. Kinsler, A.R. Frey, A.B. Coppens, J.V. Sanders, Fundamentals of Acoustics, $4^{\text {th }}$ Ed. (Wiley and Sons, New York, 2000).

34. M. Ábom, Derivation of four-pole parameters including higher order mode effects for expansion chamber mufflers with extended inlet and outlet, Journal of Sound and Vibration 137 (1990) 403-418.

35. J.B. Lawrie, I.M.M. Guled, On tuning a reactive silencer by varying the position of an internal membrane, Journal of the Acoustical Society of America 120 (2006) 780-790. 


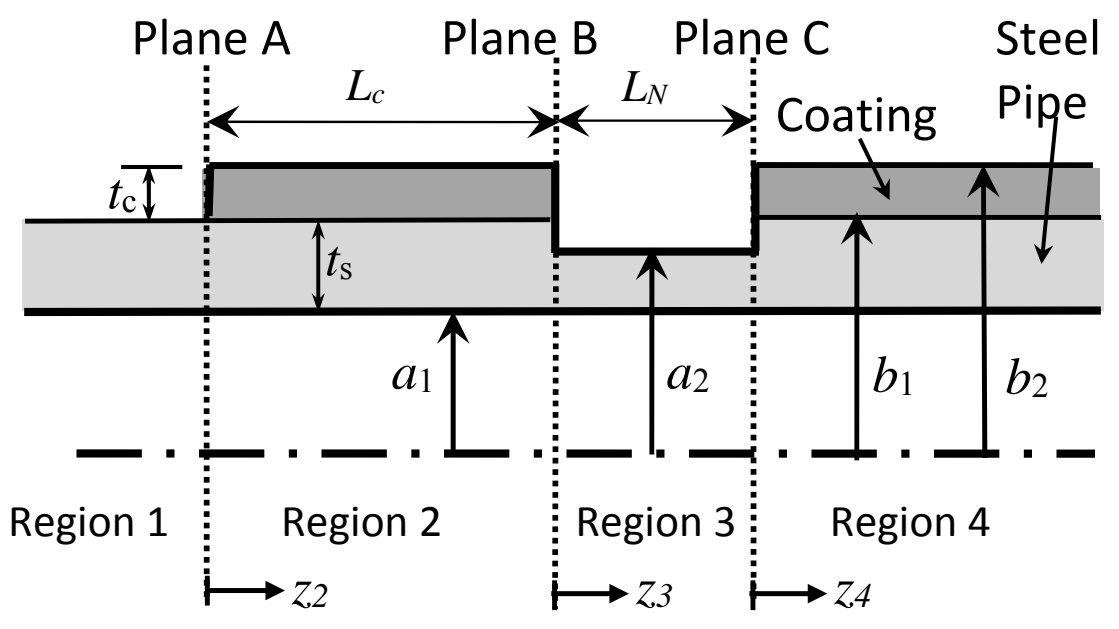

Figure 1. Geometry of uniform defect. 


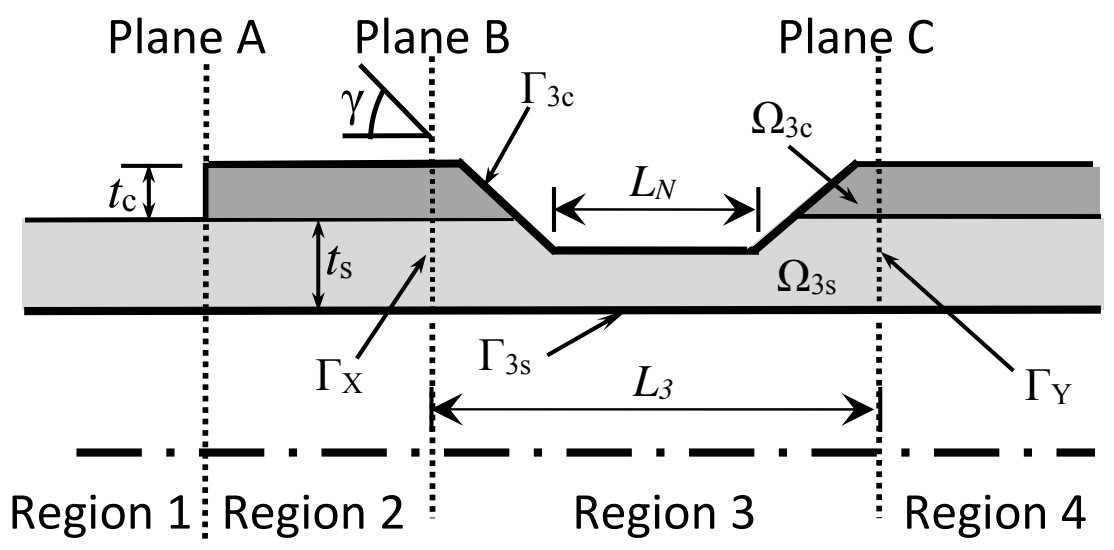

Figure 2. Geometry of non-uniform defect. 


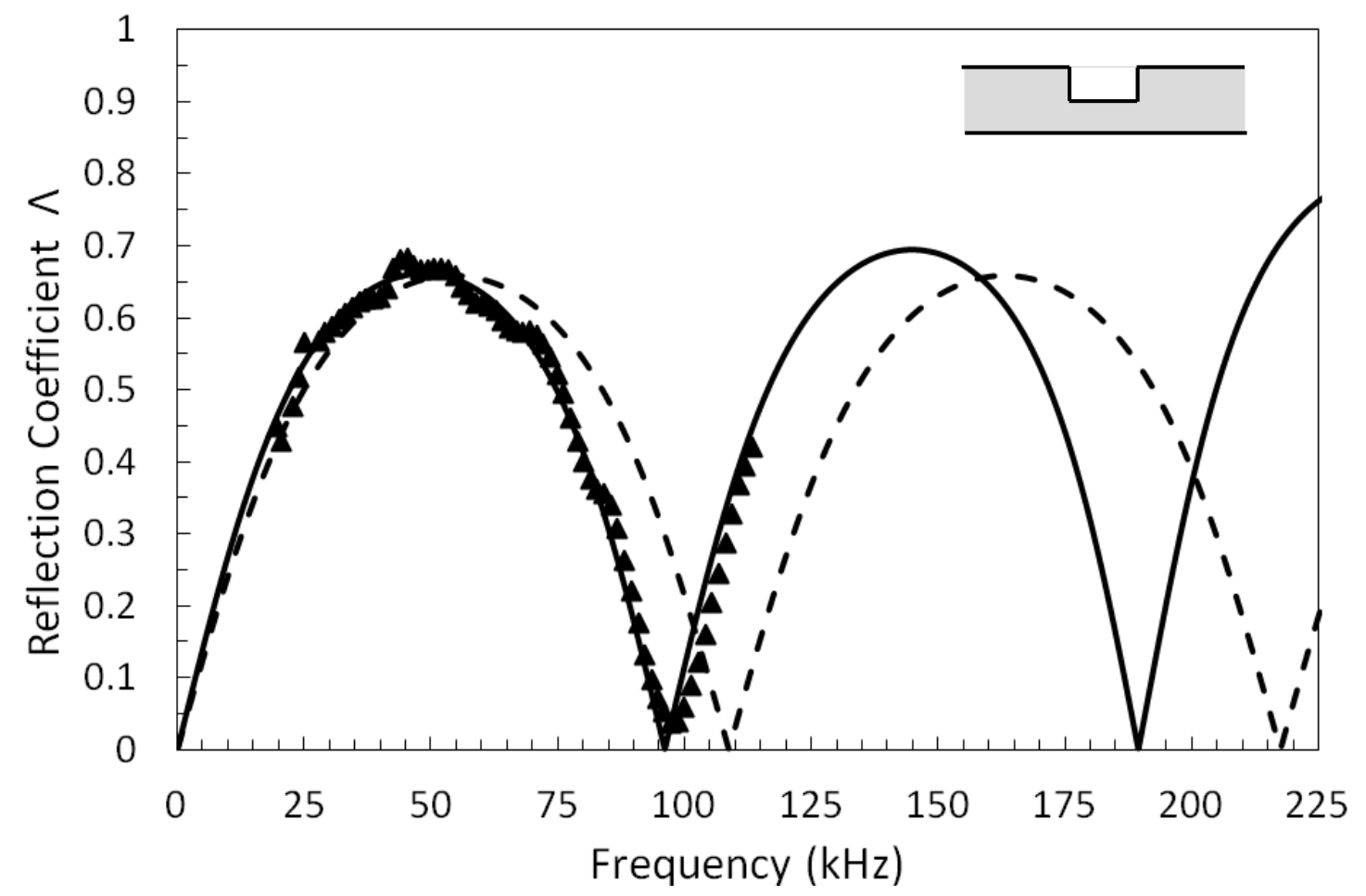

Figure 3. Reflection coefficient for uniform defect. ——, mode matching predictions; $-\mathbf{-}_{-} \quad$, plane wave predictions; $\boldsymbol{\Lambda}$, experiment. 


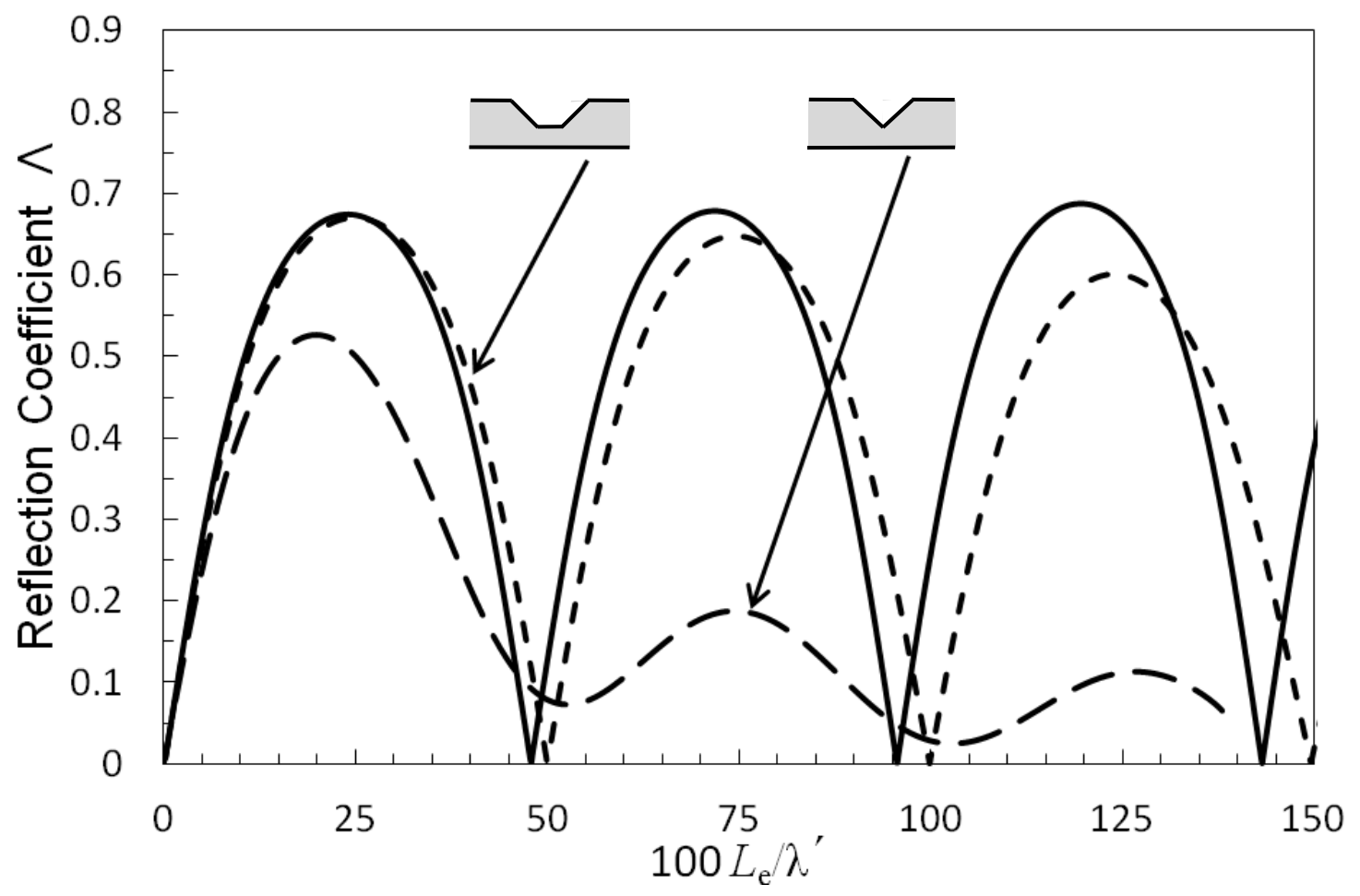

Figure 4. Hybrid method predictions for the reflection coefficient of uniform and non-uniform defects. — , uniform defect; -- , tapered defect, $\gamma=30^{\circ} ;----$, "V" defect, $\gamma=11^{\circ}$. 


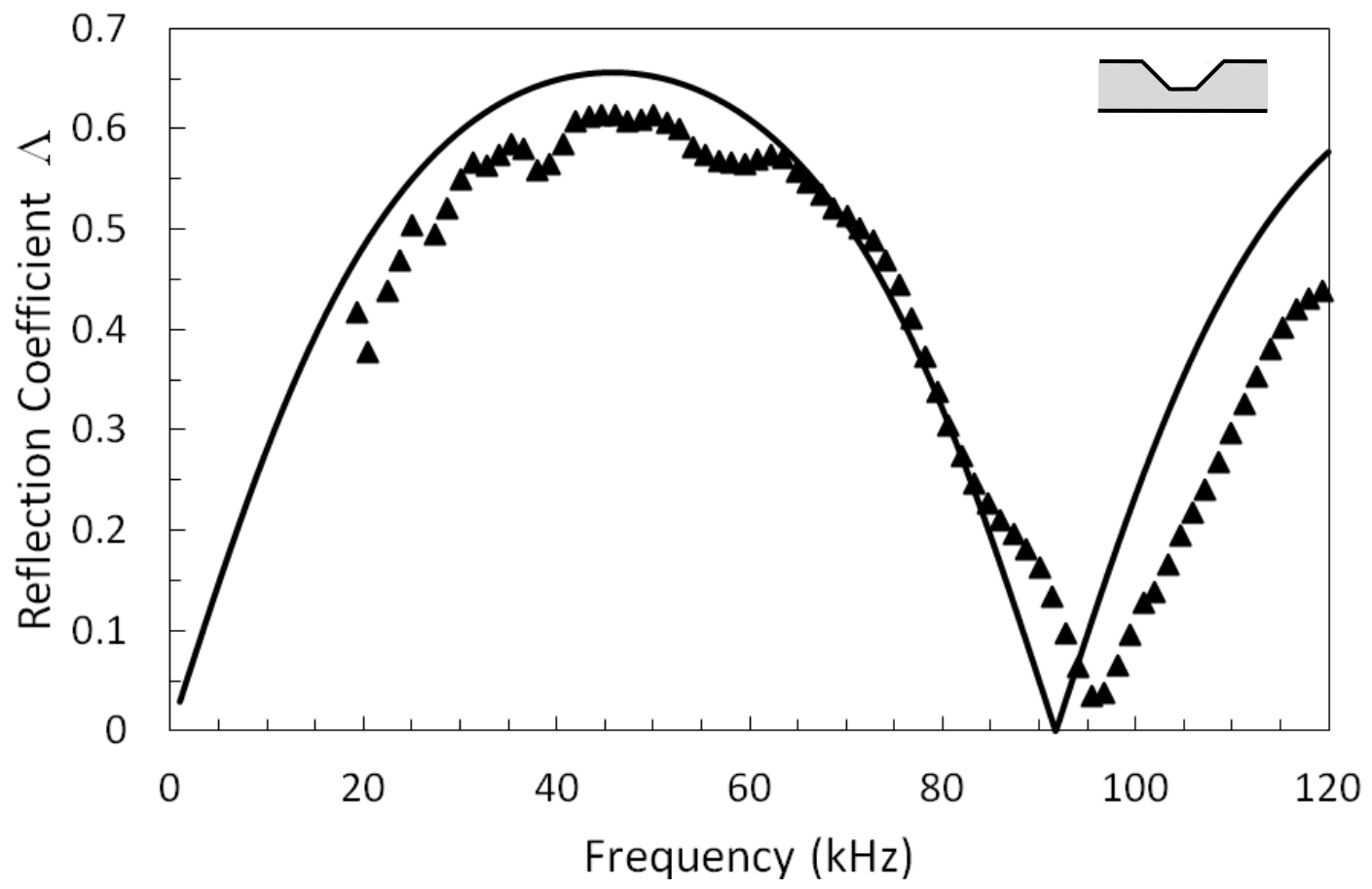

Figure 5. Reflection coefficient for tapered defect, $\gamma=30^{\circ}$. — , hybrid method predictions;

$\boldsymbol{\Delta}$, experiment. 


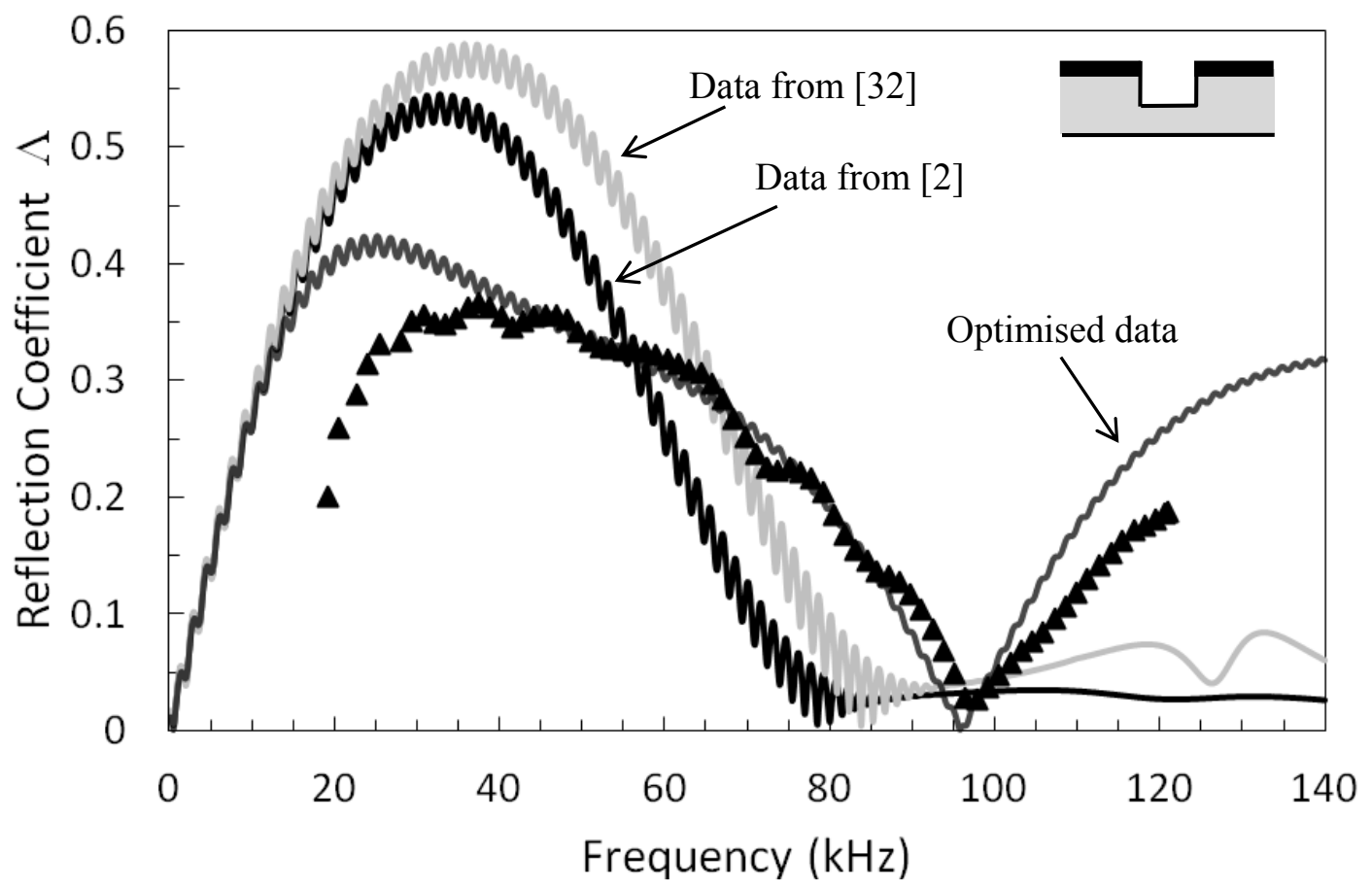

Figure 6. Mode matching predictions for the reflection coefficient of a uniform defect in a coated pipe. ——, $\widetilde{\alpha}_{c}=0.24 \times 10^{-3} \mathrm{~s} / \mathrm{m}, \rho_{c}=1500 \mathrm{~kg} / \mathrm{m}^{3}{ }_{[2] ;} \quad \widetilde{\alpha}_{c}=0.122 \times 10^{-3} \mathrm{~s} / \mathrm{m}$, $\rho_{c}=1750 \mathrm{~kg} / \mathrm{m}^{3}{ }_{[32] ;} \longrightarrow, \widetilde{\alpha}_{c}=3.9 \times 10^{-3} \mathrm{~s} / \mathrm{m}, \rho_{c}=1200 \mathrm{~kg} / \mathrm{m}^{3} ; \boldsymbol{\Lambda}$, experiment. 


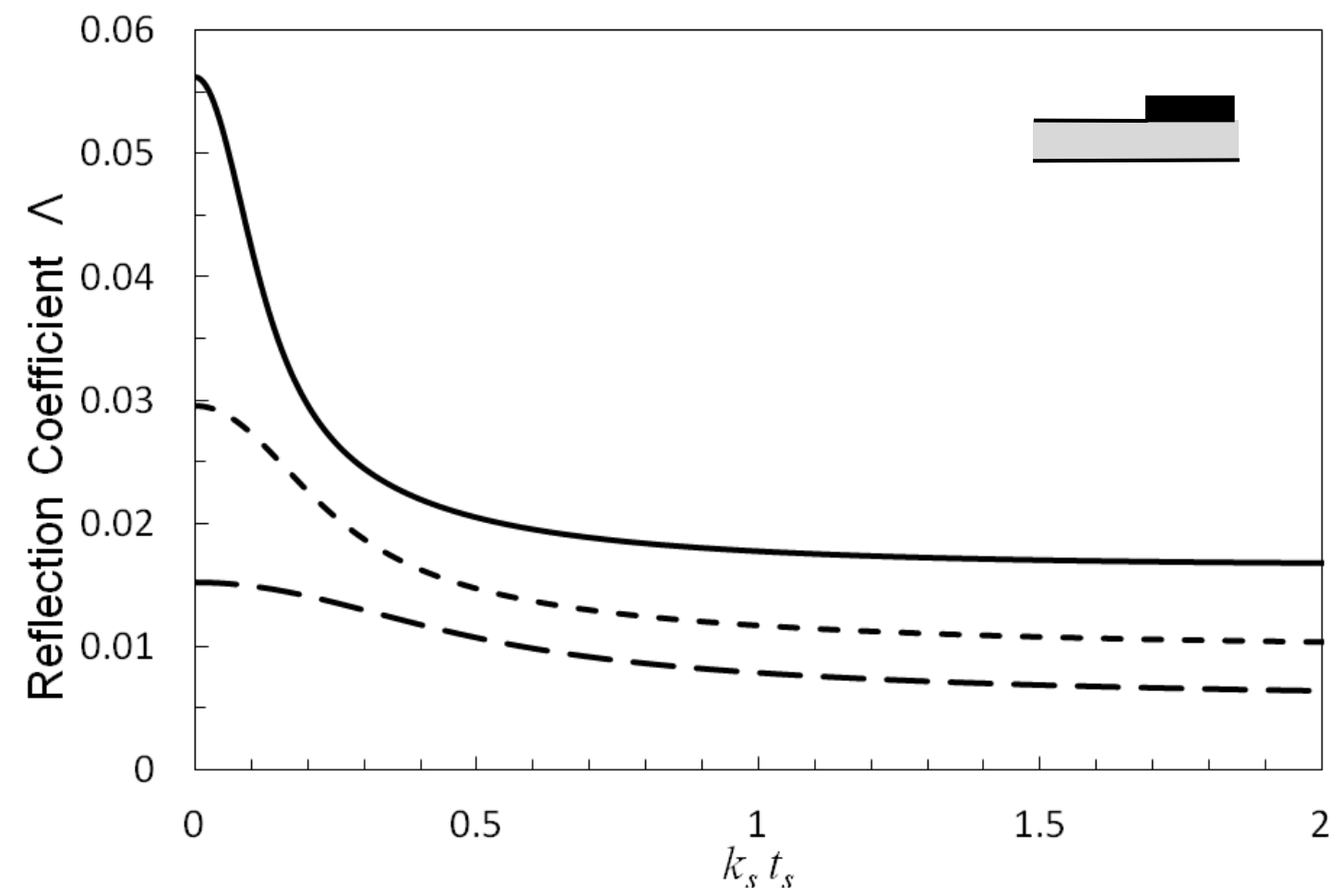

Figure 7. Mode matching predictions for the reflection coefficient of the transition between an uncoated and coated section of pipe. $\longrightarrow, t_{c} / t_{s}=1 ;----, t_{c} / t_{s}=0.5 ;--ー$, $t_{c} / t_{s}=0.25$ 


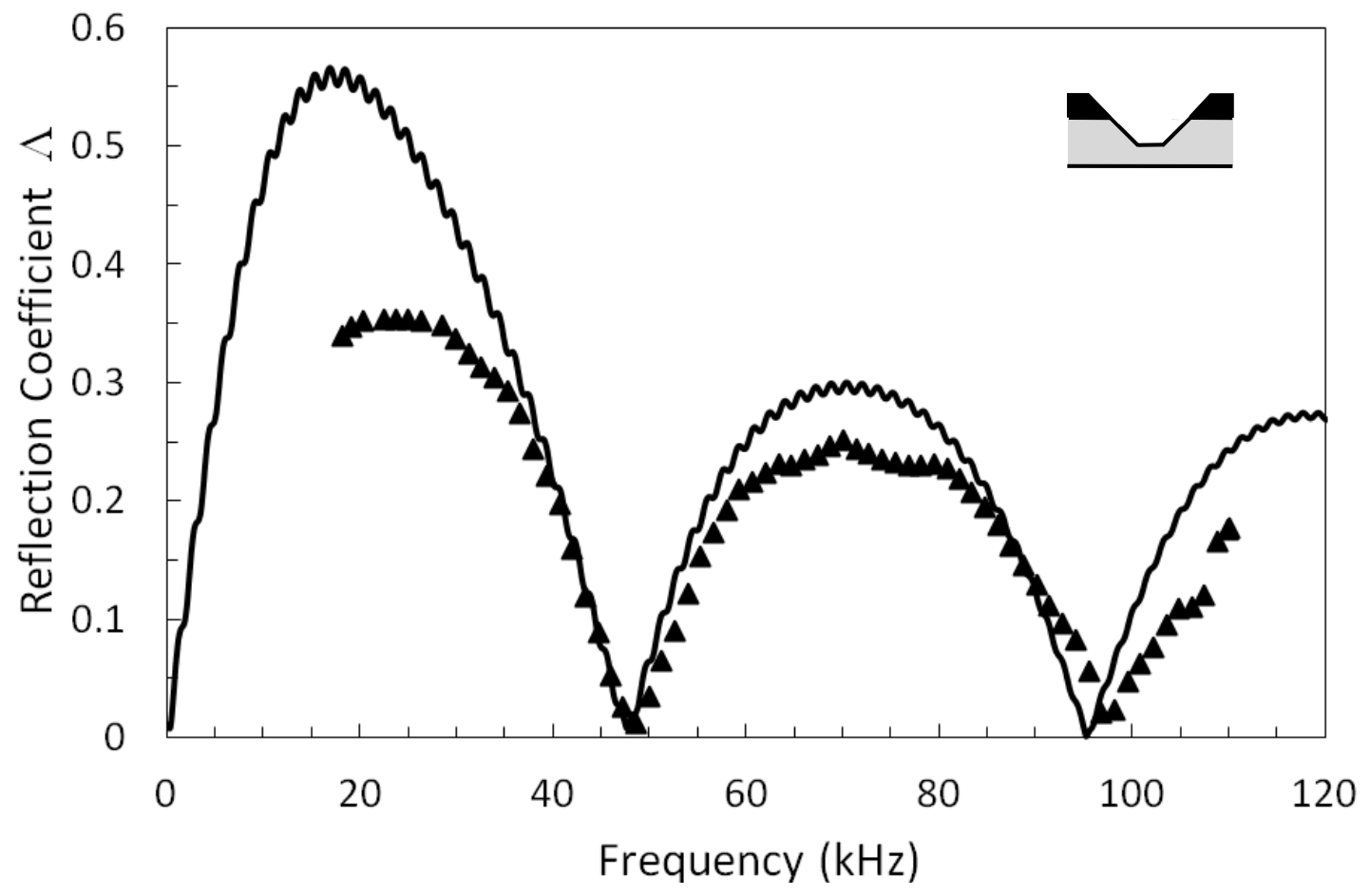

Figure 8. Reflection coefficient of a tapered defect in a coated pipe, with $\gamma=60^{\circ}$.

, hybrid method predictions; $\boldsymbol{\Delta}$, experiment. 


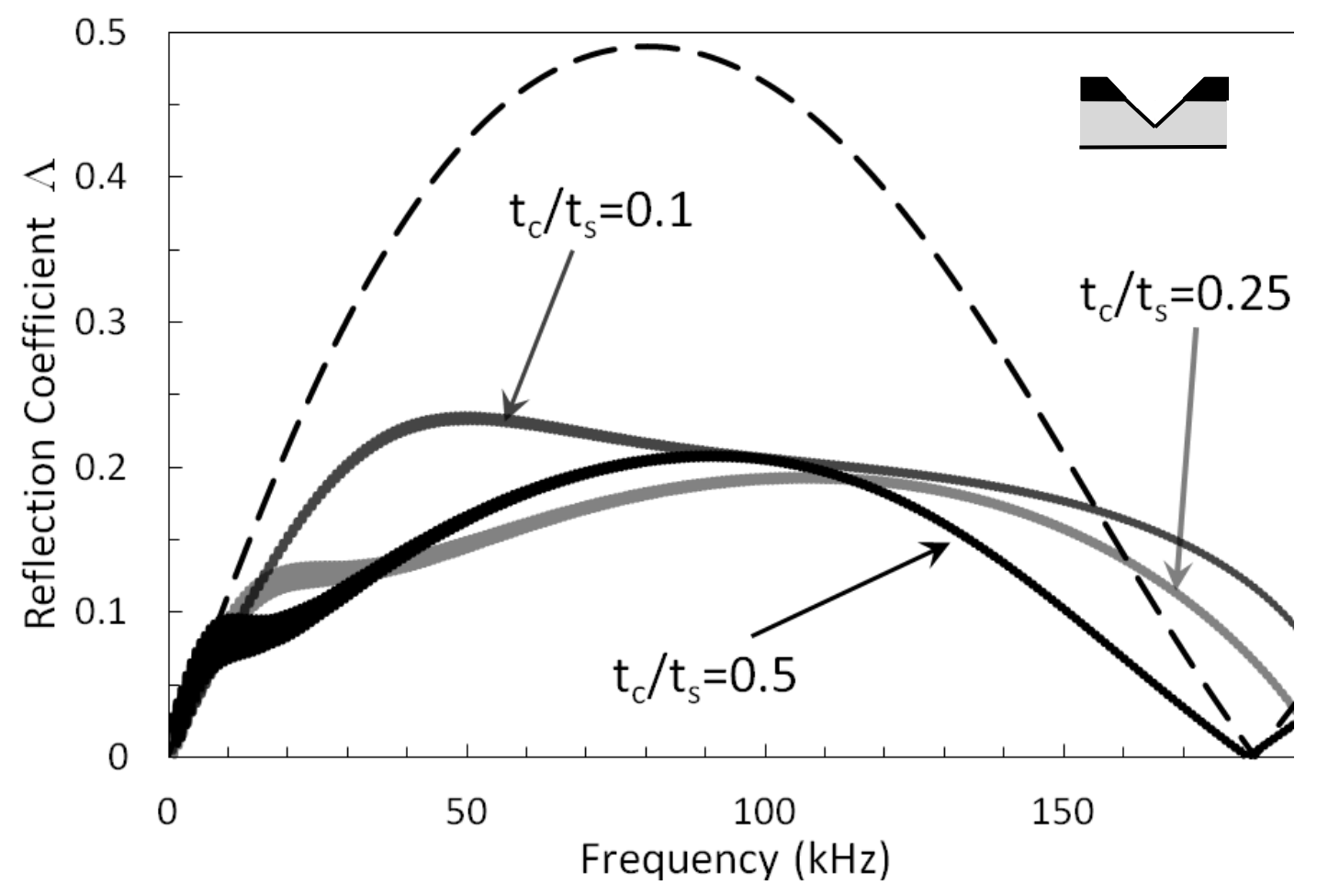

Figure 9. Hybrid method predictions for the reflection coefficient of a "V" shaped defect, with $\gamma=30^{\circ}$ and $L_{c}=2 \mathrm{~m}$. - - , uncoated pipe; $\longrightarrow, t_{c} / t_{s}=0.5$; $t_{c} / t_{s}=0.25 ;$ and $\quad t_{c} / t_{s}=0.1$ 